\title{
TTCM-Aided Rate-Adaptive Distributed Source Coding for Rayleigh Fading Channels
}

\author{
Abdulah Jeza Aljohani, Soon Xin Ng, Senior Member, IEEE, and Lajos Hanzo, Fellow, IEEE
}

\begin{abstract}
4 Abstract-Adaptive turbo-trellis-coded modulation (TTCM)5 aided asymmetric distributed source coding (DSC) is proposed, 6 where two correlated sources are transmitted to a destination 7 node. The first source sequence is TTCM encoded and is further 8 compressed before it is transmitted through a Rayleigh fading 9 channel, whereas the second source signal is assumed to be per10 fectly decoded and, hence, to be flawlessly shown at the des11 tination for exploitation as side information for improving the 12 decoding performance of the first source. The proposed scheme 13 is capable of reliable communications within $0.80 \mathrm{~dB}$ of the 14 Slepian-Wolf/Shannon (SW/S) theoretical limit at a bit error rate 15 (BER) of $\mathbf{1 0}^{-5}$. Furthermore, its encoder is capable of accom16 modating time-variant short-term correlation between the two 17 sources.
\end{abstract}

18 Index Terms-Distributed source coding (DSC), joint source19 channel coding, Slepian-Wolf (SW) coding, Turbo-trellis-oded 20 modulation (TTCM).

\section{INTRODUCTION}

${ }^{2} \mathbf{D}$ ISTRIBUTED source coding (DSC) [1] refers to the problem of compressing several physically separated, but cor24 related sources, where the receiver can perform joint decoding 25 of both encoded signals. The schematic of asymmetric DSC [2] 26 is shown in Fig. 1, where source sequence $\left\{b_{1}\right\}$ is compressed 27 before its transmission, whereas the correlated source signal $28\left\{b_{2}\right\}$ is assumed to be available at the decoder but not at the 29 source $\left\{b_{1}\right\}$. More explicitly, the encoder has to compress $\left\{b_{1}\right\}$ 30 without knowing $\left\{b_{2}\right\}$, yet the decoder is capable of exploiting 31 the knowledge of $\left\{b_{2}\right\}$ for recovering $\left\{b_{1}\right\}$.

32 More explicitly, the Slepian-Wolf (SW) [3] coding theorem 33 specifies the achievable rate regions of the compressed corre34 lated sources $\left\{b_{1}\right\}$ and $\left\{b_{2}\right\}$ for transmission to a joint decoder 35 as $R_{1} \geq H\left(b_{1} \mid b_{2}\right), R_{2} \geq H\left(b_{2} \mid b_{1}\right)$, and $R_{1}+R_{2} \geq H\left(b_{1}, b_{2}\right)$, 36 where $H\left(b_{1} \mid b_{2}\right)$ and $H\left(b_{1}, b_{2}\right)$ denote the conditional entropy 37 and joint entropy, respectively. Remarkably, this bound is 38 identical, regardless whether joint encoding or joint decoding 39 is used, i.e., regardless of where the joint processing takes

Manuscript received July 8, 2013; revised September 30, 2013; accepted October 1, 2013. This work was supported in part by the Ministry of Higher Education of Saudi Arabia, by the European Union's Seventh Framework Programme through the CONCERTO Project under Grant 288502, by the Research Councils U.K. through the India-U.K. Advanced Technology Centre, by the European Research Council under an Advanced Fellow Grant, and by the Royal Society under the Wolfson Research Merit Award. The review of this paper was coordinated by Dr. E. K. S. Au.

The authors are with the Communications, Signal Processing, and Control Research Group, School of Electronics and Computer Science, University of Southampton, Southampton SO17 1BJ, U.K. (e-mail: ajra1c09@ecs.soton. ac.uk; sxn@ecs.soton.ac.uk; 1h@ecs.soton.ac.uk).

Color versions of one or more of the figures in this paper are available online at http://ieeexplore.ieee.org.

Digital Object Identifier 10.1109/TVT.2013.2285020

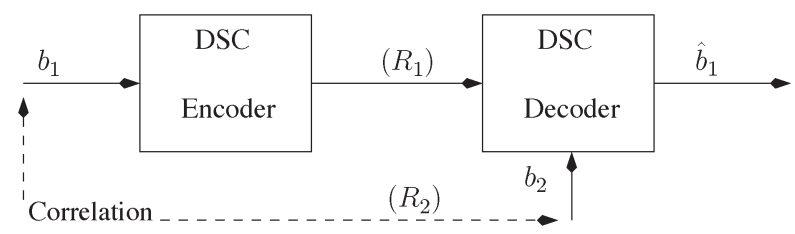

Fig. 1. Schematic of the asymmetric DSC.

place. This is quite convenient for exploiting the correlation of 40 sources, which are distant from each other with the aid of joint 41 processing at the receiver. This would facilitate for example 42 the efficient joint decoding of correlated camera-phone-video 43 sequences at the base station, namely, sequences, which portray 44 the same scene from different angles.

This promising theoretical result has led to an increasing 46 interest in a variety of applications, such as sensor networks 47 [1], robust wireless video transmission [4], and compression 48 of secure biometric data [5], where exchanging information 49 between source nodes is not possible or not practical. Applying 50 DSC techniques in wireless sensor networks, for example, 51 has led to a new processing paradigm, where the potential 52 computational complexity has been moved from the battery- 53 limited sources to the central decoder connected to the mains 54 supply.

As a consequence, the critical power constraint, which di- 56 rectly predetermines the life span of the wireless node, is 57 fulfilled [1]. The first practical DSC technique was proposed 58 in [6], where both sources $\left\{b_{1}\right\}$ and $\left\{b_{2}\right\}$ are assumed to be 59 emitting equiprobable codewords, but they exhibit a difference 60 because $\left\{b_{2}\right\}$ is known only to the joint decoder but not to the 61 encoder of $\left\{b_{1}\right\}$. Naturally, this assumption does not preclude 62 that the codewords of $\left\{b_{2}\right\}$ actually received from a remote 63 source, but they must be first perfectly recovered in isolation 64 before they may be used by the joint decoder for recovering 65 $\left\{b_{1}\right\}$. Then, the codewords of both sources are grouped in 66 cosets, where the members of each coset are separated by 67 the maximum possible Hamming distance. Given $\left\{b_{2}\right\}$ at the 68 receiver, it is sufficient to transmit the index of the specific coset 69 hosting the codewords of $\left\{b_{1}\right\}$. The decoder then estimates the 70 transmitted codeword by choosing the one that is closest to the 71 side information constituted by $\left\{b_{2}\right\}$ of a given coset in terms 72 of the Hamming distance.

The idea of using channel coding techniques ${ }^{1}$ has enabled 74 practical solutions to be developed. Practical Slepian-Wolf 75

\footnotetext{
${ }^{1}$ Since the correlation between the sources may be interpreted as the ameliorating effect of a "virtual" channel, a good channel code having, for example, a maximum minimum Hamming distance will be a good SW code [7].
} 
76 schemes using turbo codes (TCs) were proposed for example 77 in [2], [8], and [9], whereas low-density parity-check (LDPC) 78 codes were considered in [10]-[12]. However, finding the best 79 code for approaching the Slepian-Wolf/Shannon (SW/S) limit 80 was not considered in [2], [8], and [9]. Later, a so-called "super" $81 \mathrm{TC}$ was proposed in [13], aiming for approaching the SW/S 82 limit, when communicating over additive white Gaussian noise 83 (AWGN) channels. However, the scheme proposed in [13] for 84 an AWGN channel suffers from an error floor when communi85 cating over Rayleigh fading channels that makes the system less 86 suitable for wireless applications. A modified LDPC code was 87 proposed in [14] for mitigating the error floor, but nonetheless, 88 a high error floor persists when the correlation between the 89 sources is low. A joint turbo equalizer and decoder scheme 90 was proposed for asymmetric DSC in [15], whereas an iterative 91 joint turbo equalizer and decoder scheme was conceived for 92 transmission over a multipath Rayleigh fading multiple-access 93 channel in [16]. Both schemes have achieved a near-SW/S 94 performance, albeit at high joint decoding complexity. More 95 specifically, 35 iterations were invoked between the decoder 96 components in [15], whereas as many as 350 iterations were 97 required in [16] for attaining a near-SW/S performance. By 98 contrast, we only invoke eight turbo iterations in our TTCM 99 decoder, where both constituent decoders have comparable 100 complexity [17].

101 Furthermore, in practice, the short-term correlation among 102 the sources might be time variant; hence, adaptive-rate schemes 103 have to be considered, where the code rate is controlled via 104 a feedback channel. More specifically, if the bit error rate 105 (BER) evaluated after decoding exceeds a given threshold, 106 more syndromes (or parity bits if parity puncturing is used) 107 will be requested from the transmitter. A pair of innovative 108 adaptive-rate LDPC schemes was proposed in [18], whereas 109 adaptive-rate TCs were designed in [19]. In [18], the encoder 110 stored the syndromes and incrementally transmitted them to 111 the receiver, when the decoder failed to find the legitimate 112 codeword. Both papers considered an asymmetric DSC struc113 ture based on the puncturing of the syndrome generated by the 114 channel encoders, while stipulating the idealized simplifying 115 assumption of modeling the channel as the parallel combination 116 of a perfect channel and a binary symmetric channel (BSC). 117 More advanced adaptive-rate schemes considered the employ118 ment of a polar code [20] or efficient particle-based belief119 propagation-aided decoding [21] and density-evolution-based 120 decoding techniques [18].

121 Against this background, we propose a novel bandwidth122 efficient turbo-trellis-coded modulation (TTCM) scheme, 123 which combines the functions of coding and modulation for 124 conceiving a new DSCs system. TTCM [22] has a structure 125 similar to that of the family of binary TCs, where two identical 126 parallel-concatenated TCM schemes rather than conventional 127 codes are employed as component codes. The classic TTCM 128 design was outlined in [22], which is based on the search for 129 the best TCM component codes using the so-called "punctured" 130 minimal distance criterion, to approach the capacity of the 131 AWGN channel. The TTCM code advocated was designed to 132 improve the attainable throughput by considering the design of 133 error-correcting code and modulation where the parity bits are absorbed in without any bandwidth expansion by increasing 134 the number of bits per modulated symbol. By contrast, all 135 separated channel codes, such as turbo or LDPC codes, impose 136 a bandwidth expansion, which is proportional to the code rate. 137 Furthermore, a novel adaptive-rate mechanism is conceived for 138 increasing the system's effective throughput, while ensuring an 139 infinitesimally low BER. Hence, our new contributions are as 140 follows.

- We propose a uniquely amalgamated DSC and TTCM 142 (DSTTCM) scheme for SW coding, which is capable of 143 attaining a near-SW/S performance for a wide range of 144 source correlation values. Our new scheme exhibits no 145 error floor, ${ }^{2}$ despite its low complexity. Furthermore, a 146 carefully constructed modified symbol-based maximum 147 a posteriori (MAP) algorithm is conceived for exploiting 148 the side information available at the decoder. Additionally, 149 we eliminate the aforementioned idealized simplifying 150 assumptions exploited in the prior literature [18], [19], 151 [23], and consider more realistic uncorrelated Rayleigh 152 fading channels and BSCs.

- Furthermore, adaptive DSTTCM (A-DSTTCM) is pro- 154 posed for accommodating the near-instantaneously time- 155 varying nature of the wireless channel and the short-term 156 correlation fluctuations among the sources. More explic- 157 itly, the system adapts its parameters according to both the 158 channel quality and the correlation $\rho$ of the sources, ${ }^{3}$ while 159 maintaining a given target BER.

The remainder of this paper is organized as follows. The 161 proposed system model is described in Section II. The proposed 162 scheme is designed in Section III. Section IV discusses our 163 results. Finally, our conclusions are offered in Section V.

\section{SYSTEM MODEL}

165

The asymmetric DSC scenario in Fig. 1 was considered here, 166 where sequence $\left\{b_{2}\right\}$ is transmitted at the rate of $R_{2}=H\left(b_{2}\right), 167$ which is typically referred to as "side information" in most 168 contributions [2], [8]; again, however, it can be also interpreted 169 as another desired source signal, which was perfectly recovered. 170 Sequence $\left\{b_{2}\right\}$ can be also transmitted through an independent 171 Rayleigh-fading channel, in which case, a similar encoder 172 structure to that of the first source $\left\{b_{1}\right\}$ has to be implemented/ 173 The problem in this scenario may be considered a symmetric 174 DSC one. Symmetric DSC compression was discussed, for 175 example, in [13], [14], and [16], but due to space limitations, 176 it is beyond the scope of this paper.

Furthermore, crossover probability $p_{e}$ used for modeling 178 correlation parameter $\rho$ may be assumed to be the overall proba- 179 bility of error, which is denoted as $p_{e_{1}}$ and $p_{e_{2}}$. More explicitly, 180 the error between the two sources is denoted by $p_{e_{1}}$ and $p_{e_{2}} 181$ which characterizes the transmission error between the second 182 source and the destination. Thus, in this scenario, the overall 183 error obeys $p_{e} \leq p_{e_{1}}+p_{e_{2}}$. The correlated sequence $\left\{b_{1}\right\}$ is 184 then compressed for approaching the Slepian-Wolf bound to a 185

\footnotetext{
${ }^{2}$ As documented in Fig. 4, no error floor is observed at above BER level of $10^{-6}$; hence, our scheme is evidently suitable for wireless applications.

${ }^{3} \rho$ is expressed using a crossover probability $p_{e}$ as $\rho=1-2 p_{e}$.
} 


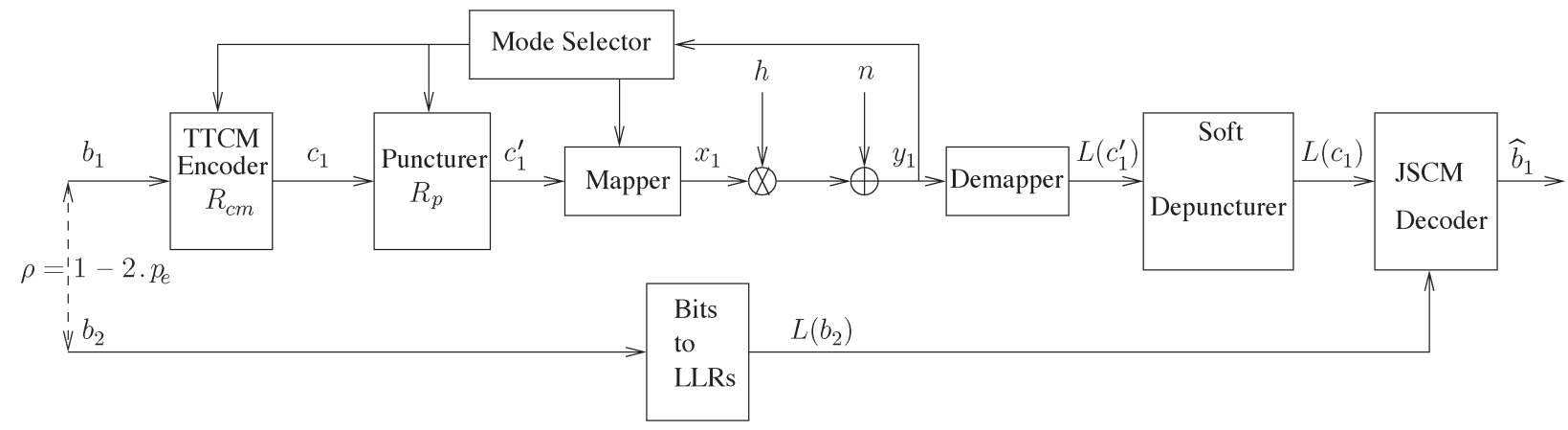

Fig. 2. Block diagram of the A-DSTTCM system communicating over Rayleigh fading channels. Sources $\left\{b_{1}\right\}$ and $\left\{b_{2}\right\}$ are assumed to be correlated, i.e., we have $H\left(b_{1} \mid b_{2}\right) \neq H\left(b_{1}\right)$, and $L(\cdot)$ denotes the LLRs.

186 rate of $R_{1}=H\left(b_{1} \mid b_{2}\right)$ to achieve the overall rate of $H\left(b_{1}, b_{2}\right)$. 187 Typically, the BSC is used for modeling the correlation between 188 the two source sequences $\left\{b_{1}\right\}=\left\{b_{1}^{1}, b_{1}^{2}, \ldots, b_{1}^{i}, \ldots, b_{1}^{N}\right\}$ and $189\left\{b_{2}\right\}=\left\{b_{2}^{1}, b_{2}^{2}, \ldots, b_{2}^{i}, \ldots, b_{2}^{N}\right\}$, where $N$ is the length of each 190 source block.

191 For example, when $\left\{b_{1}\right\}$ and $\left\{b_{2}\right\}$ have a correlation of $192 \rho=0.9$ given the perfect knowledge of $\left\{b_{2}\right\},\left\{b_{1}\right\}$ may be in193 terpreted as the output signal of BSC, which was contaminated 194 by the bit-flipping error events occurring with a probability 195 of $p_{e}$. Source sequence $\left\{b_{1}\right\}$ is generated by an equiprobable 196 binary symmetric independent identically distributed (i.i.d.) 197 source, whereas $\left\{b_{2}\right\}$ can be defined as $b_{1}^{i}=b_{2}^{i} \oplus e_{i}$, where $\oplus$ 198 is the modulo- 2 addition operation, and $e_{i}$ is an independent 199 binary random variable assuming the logical value of 1 with 200 a crossover probability of $p_{e}$ and of 0 with a probability of $201\left(1-p_{e}\right)$. Both the random variables of $b_{1}^{i}$ and $b_{2}^{i}$ in the pair 202 of bit streams $\left\{b_{1}\right\}$ and $\left\{b_{2}\right\}$ may be assumed to be i.i.d. for 203 the bit index $i$; hence, both sources emit equiprobable [16] bits. 204 Consequently, the entropy of each source is unity, which yields 205 conditional entropy of

$$
\begin{aligned}
H\left(p_{e}\right) & =H\left(b_{1} \mid b_{2}\right) \\
& =\lim _{i \rightarrow \infty} \frac{1}{i} H\left[\left(\left(b_{1}^{1}, \cdots, b_{i}^{1}, \cdots b_{N}^{1}\right) \mid\left(b_{1}^{2}, \cdots, b_{i}^{2}, \cdots b_{N}^{2}\right)\right)\right]
\end{aligned}
$$

206 where $H\left(p_{e}\right)=p_{e} \log _{2}\left(1 / p_{e}\right)+\left(1-p_{e}\right) \log _{2}\left(1 / 1-p_{e}\right)$ is 207 the entropy of the binary random variable, and $e_{i}$ is used for 208 parametrizing the side information/ Therefore, the achievable $209 \mathrm{SW}$ rate region is given by the following three inequalities [16]:

$$
\begin{gathered}
R_{1} \geq H\left(p_{e}\right) \\
R_{2} \geq H\left(p_{e}\right) \\
R_{1}+R_{2} \geq 1+H\left(p_{e}\right) .
\end{gathered}
$$

210 Let us now embark on the design of a joint source-channel 211 (JSC) decoding scheme for minimizing the signal-to-noise 212 power ratio (SNR) required for approaching the SW/S bound, 213 while maintaining reliable communications. More explicitly, 214 let us define $\mathrm{SNR}=R_{1} \cdot E_{b} / N_{0}$, where $E_{b} / N_{0}$ denotes the 215 energy per bit to noise power spectral density.

\section{A. Encoder}

The block diagram of the proposed A-DSTTM scheme con- 217 sidered for transmitting correlated sources is shown in Fig. 2, 218 where $L(\cdot)$ denotes the log-likelihood ratios (LLRs) of the bits. 219 As shown in Fig. 2, the input sequence $\left\{b_{1}\right\}$ is fed into a 220 TTCM encoder, which has a coding rate of $R_{\mathrm{cm}}=m / m+1221$ and invokes a $2^{m+1}$-level modulation scheme. The TTCM- 222 encoded bits are then punctured at a rate of $R_{p}$. The resultant bit 223 sequence $\left\{c_{1}^{\prime}\right\}$ is then mapped to the corresponding modulated 224 symbols $\left\{x_{1}\right\}$ before their transmission over an uncorrelated 225 Rayleigh fading channel. The second bit sequence $\left\{b_{2}\right\}$ will 226 be converted to the LLRs $L\left(b_{2}\right)=L_{e}$, which is then will be 227 exploited as side information. This conversion is necessary 228 because the joint decoder is a soft-decision-based one. These 229 LLRs are characterized by the aforementioned crossover prob- 230 ability $p_{e}$ and can be estimated as

$$
\begin{aligned}
L\left(b_{2} \mid b_{1}\right) & =\ln \left[\frac{\operatorname{Pr}\left(b_{2}=+1 \mid b_{1}\right)}{\operatorname{Pr}\left(b_{2}=-1 \mid b_{1}\right)}\right] \\
& =\ln \left[\frac{\left(1-p_{e}\right) \operatorname{Pr}\left(b_{1}=+1\right)+p_{e} \operatorname{Pr}\left(b_{1}=+1\right)}{\left(1-p_{e}\right) \operatorname{Pr}\left(b_{1}=-1\right)+p_{e} \operatorname{Pr}\left(b_{1}=-1\right)}\right] .
\end{aligned}
$$

We assume that these LLRs are available at the destination 232 and to be exploited by the joint decoder, whereas $p_{e}$ can be 233 estimated at the decoder using (11).

As an example, we use a rate $R_{\mathrm{cm}}=1 / 2 \mathrm{TTCM}$ encoder 235 relying on a puncturer of rate $R_{p}=2 / 1$, which punctures 236 one bit out of two encoded bits. We assume that all the 237 systematic bits are punctured, whereas all the parity bits are 238 transmitted to the decoder. Hence, the overall code rate is 239 $R_{1}=R_{\mathrm{cm}} \cdot R_{p}=1$. However, the parity bit sequence may be 240 also further punctured to achieve an increased compression 241 ratio. The resultant bits are then mapped to binary phase-shift 242 keying (BPSK) symbols, i.e., the modulation mode has been 243 changed from quadrature phase-shift keying (QPSK) to BPSK 244 (QPSK/BPSK). Thus, the corresponding effective throughput 245 is given by $\eta=R_{1} \cdot \log _{2}(2)=1$ bits per symbol (BPS). Then, 246 the modulated symbol sequence $\left\{x_{1}\right\}$ is transmitted over an 247 uncorrelated Rayleigh fading channel, and the received symbol 248 $y_{1}$ is given by

$$
y_{1}=h x_{1}+n
$$




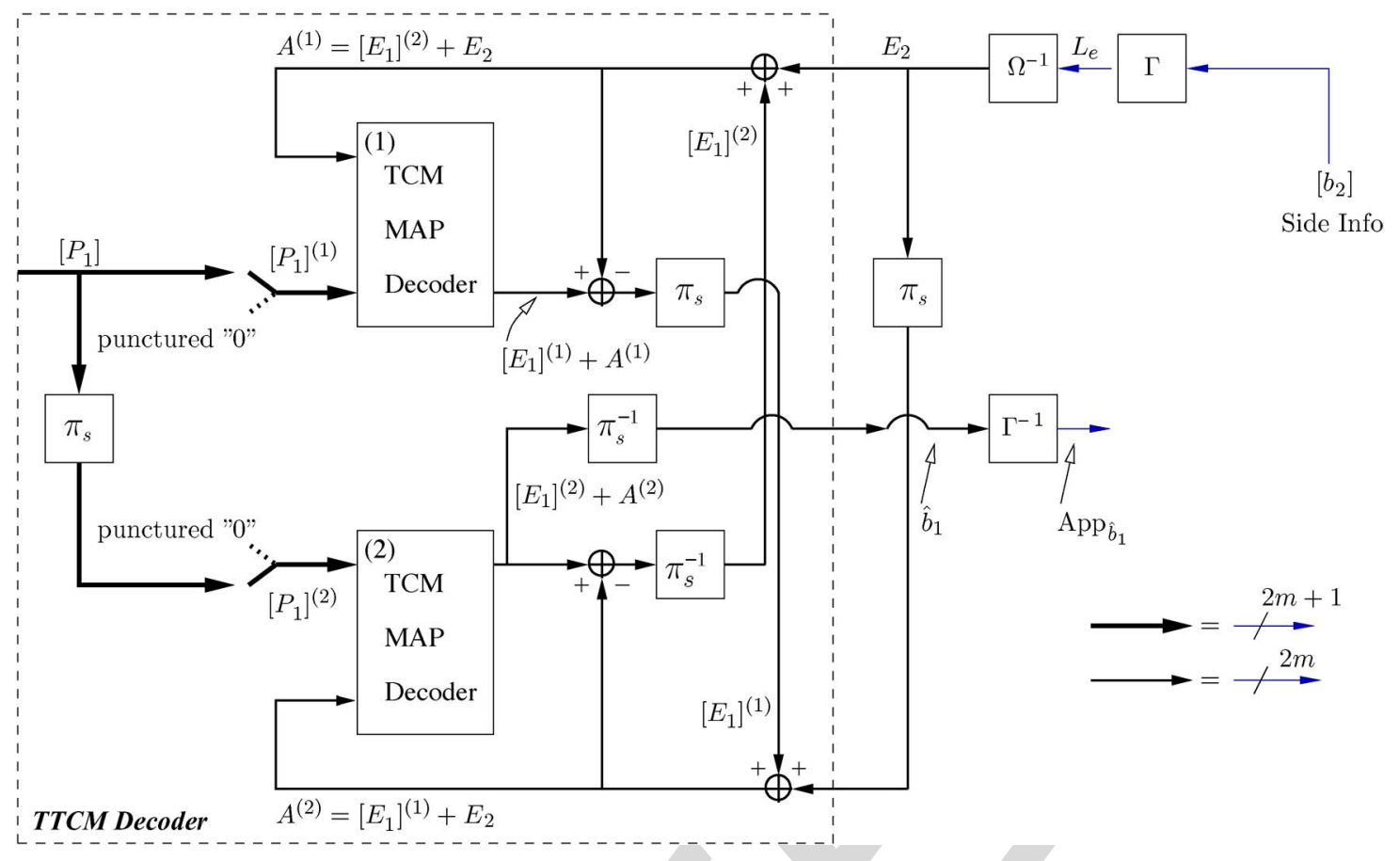

Fig. 3. Block diagram of the JSC-aided TTCM decoder conceived for our DSC system. Notations $\pi_{s}$ and $\pi_{s}^{-1}$ represent the symbol interleaver and deinterleaver, respectively, whereas $\Gamma$ and $\Gamma^{-1}$ denote the relevant symbol-to-LLR and LLR-to-symbol probability conversion.

250 where $h$ is the fading coefficient of the channel, and $n$ is the 251 AWGN having a variance of $N_{0} / 2$ per dimension. The short252 term average received SNR is given by

$$
\mathrm{SNR}_{r}=\frac{E\left\{|h|^{2}\right\} E\left\{\left|x_{1}\right|^{2}\right\}}{N_{0}}
$$

253 where $x_{1}$ represents the modulated symbols of source $\left\{b_{1}\right\}$ 254 after puncturing. Furthermore, we have $E\left\{\left|x_{1}\right|^{2}\right\}=1$, and 255 the SNR of $\gamma_{r}=10 \log _{10}\left(|h|^{2} / N_{0}\right)[\mathrm{dB}]$ is estimated for each 256 received block. Note that $\left\{b_{2}\right\}$ is related to $\left\{b_{1}\right\}$ according 257 to $b_{1}^{i}=b_{2}^{i} \oplus e_{i}$, as detailed in Section II. Diverse effective 258 throughputs may be derived by changing $R_{\mathrm{cm}}$ and $R_{p}$; hence, 259 the proposed scheme exhibits substantial flexibility. In our 260 A-DSTTCM scheme, the following modes are chosen at the 261 encoder to ensure that BER $<10^{-5}$ :

262 - No transmission;

263 - DSTTCM-QPSK/BPSK;

264 - DSTTCM-8PSK/QPSK;

265 - DSTTCM-16-quadrature amplitude modulation (QAM)/ 266 8PSK;

267 - DSTTCM-32QAM/16QAM.

268 Thus, the effective throughput of our adaptive system as269 sumes the values of $\eta=\{0,1,2,3,4\}$ BPS.

\section{B. Joint Source-Channel Decoder}

271 Our JSC-decoding-aided DSTTCM scheme is shown in 272 Fig. 3. Both TCM decoders invoke the symbol-based MAP 273 algorithm [17] operating in the logarithmic domain. The TCM 274 decoders are labeled with the round-bracketed indexes, whereas 275 the notation $P, A$, and $E$ denote the logarithmic probabilities 276 of the a posteriori, a priori, and extrinsic information, respec277 tively, where $L_{e}=L\left(b_{2} \mid b_{1}\right)$. The $2^{(m+1)}$-ary $P$ probabilities associated with a specific $(m+1)$-bit TTCM-coded symbol 278 $\left\{c_{1}^{\prime}\right\}$ are fed into the TTCM MAP decoder. A pair of signal 279 components is generated by the constituent TCM decoders [17]; 280 specifically, the extrinsic probability $E$ is generated by each 281 of the TCM decoders, whereas the a priori probability $A$ is 282 gleaned by each TCM decoder from the other one. Furthermore, 283 as shown in Fig. 3, the additional extrinsic probability $E_{2} 284$ extracted from the side information $\left\{b_{2}\right\}$ is also added to 285 the a priori probability $A$, yielding $A^{(1,2)}=\left[E_{1}\right]^{(2,1)}+E_{2} .286$ Each of the constituent TCM blocks in Fig. 3 calculates the 287 a posteriori probabilities using the forward and backward re- 288 cursion methods. ${ }^{4}$

Upon recalling (4), we are now in the position to formulate 290 the channel's transition metric as

$$
\eta_{i}(\grave{s}, s)=\ln \left\{\frac{1}{\pi N_{0}} \mathrm{e}^{-\left|y_{1}-h x_{1}\right|^{2} / N_{0}}\right\} .
$$

Then, both the backward and forward recursion methods of 292 [17] are invoked for calculating $\beta_{i-1}(\grave{s})$ and $\alpha_{i}(s)$ as follows: 293

$$
\begin{aligned}
\alpha_{i}(s) & =\max _{\operatorname{all} \grave{s}}^{*}\left(\alpha_{i-1}(\grave{s})+\eta_{i}(\grave{s}, s)+A^{(1,2)}\right) \\
\beta_{i-1}(\grave{s}) & =\max _{\text {all } s}^{*}\left(\beta_{i}(s)+\eta_{i}(\grave{s}, s)+A^{(1,2)}\right)
\end{aligned}
$$

where $\max ^{*}$ represents the Jacobian logarithm [17] evaluating 294 all variables in the logarithmic domain, with $(\grave{s}, s)$ denoting the 295 transitions emerging from the previous state $\grave{s}$ to the present 296 state $s$. 


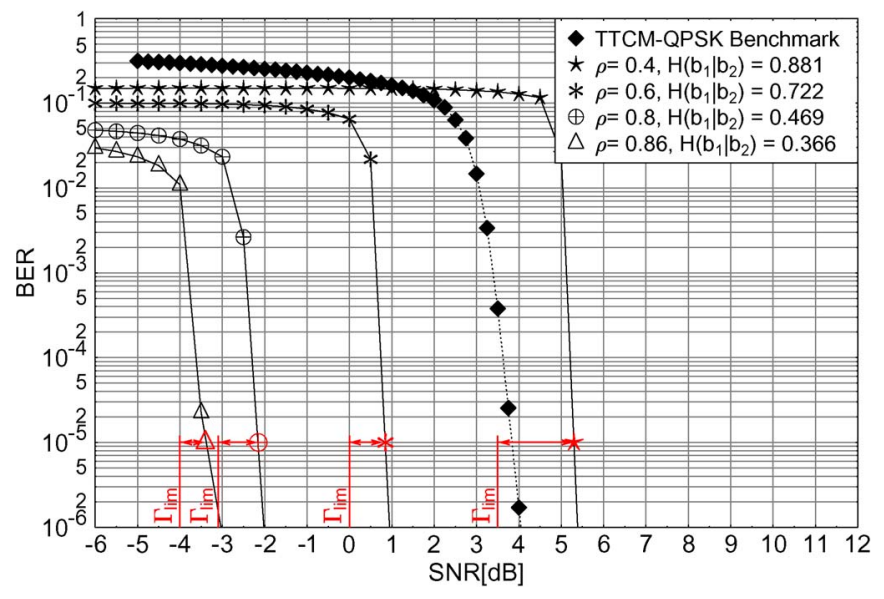

Fig. 4. BER versus SNR performance of the proposed DSTTCMQPSK/BPSK scheme for correlation parameters of $\rho=\{0.4,0.6,0.8,0.86\}$ when transmitting over uncorrelated Rayleigh fading channels. The number of decoding iterations is $I=8$.

\section{RATE REgION DESIGN AND ANALYSiS}

299 The achieved rate region experienced in a noisy channel for 300 both sources is given by [13], [16]:

$$
\begin{aligned}
H\left(b_{1}, b_{2}\right) & \leq \frac{C_{1}}{R_{1}}+\frac{C_{2}}{R_{2}} \\
& \leq \frac{1}{R_{1}} E\left\{\log _{2}\left(1+\gamma_{1}\right)\right\}+\frac{1}{R_{2}} E\left\{\log _{2}\left(1+\gamma_{2}\right)\right\}
\end{aligned}
$$

301 where $C_{1}=E\left\{\log _{2}\left(1+\gamma_{1}\right)\right\}$ and $C_{2}=E\left\{\log _{2}\left(1+\gamma_{2}\right)\right\}$ de302 note the ergodic channel capacities between each of the sources 303 and the destination, whereas $\gamma_{1}$ and $\gamma_{2}$ denote the correspond$304 \mathrm{ing}$ received SNRs. In our asymmetric system, we assume 305 that $\left\{b_{2}\right\}$ is transmitted at $R_{2}=H\left(b_{2}\right)=1$, whereas we aim 306 for compressing $\left\{b_{1}\right\}$ to its minimum rate, namely, to $R_{1}=$ $307 H\left(b_{1} \mid b_{2}\right)$. Then, based on (9), the effective throughput of our 308 scheme for the $\left\{b_{1}\right\}$ link can be expressed as $\eta_{S W}=R_{1}$. $309 H\left(b_{1} \mid b_{2}\right)$, whereas the SW/S bound is calculated as [15]

$$
R_{1} \cdot H\left(b_{1} \mid b_{2}\right) \leq C_{1}
$$

310 where $C_{1}$ represents the ergodic capacity of the uncorrelated 311 Rayleigh fading channel.

312 First we characterize the BER performance of our DSTTCM313 QPSK/BPSK scheme employing a range of correlations $\rho=$ $314\{0.4,0.6,0.8,0.86\}$. We opted for using 1/2-rate TTCM for 315 encoding a block of $N_{S}=12000$ symbols, resulting in $N_{b}=$ 31624000 bits before we remove all of the systematic bits from 317 the TTCM-coded sequence with the aid of puncturing. The 318 BER versus SNR performance of the proposed system is shown 319 in Fig. 4. Note that the SNR can be calculated in decibels 320 as $\operatorname{SNR}(\mathrm{dB})=E_{b} / N_{0}(\mathrm{~dB})+10 \log \left(R_{1}\right)$. The minimum SNR $321 \Gamma_{\lim }$ required for approaching the SW/S bound can be inferred 322 in Fig. 5, which shows both the continuous-input-continuous323 output memoryless channel's (CCMC) capacity and the 324 corresponding BPSK-based discrete-input-continuous-output 325 memoryless channel's (DCMC) capacity curve. For example, 326 when aiming for a target throughput of $\eta_{S W}=0.366$ BPS for 327 our DSTTCM-QPSK/BPSK scheme, the DCMC curve indi-

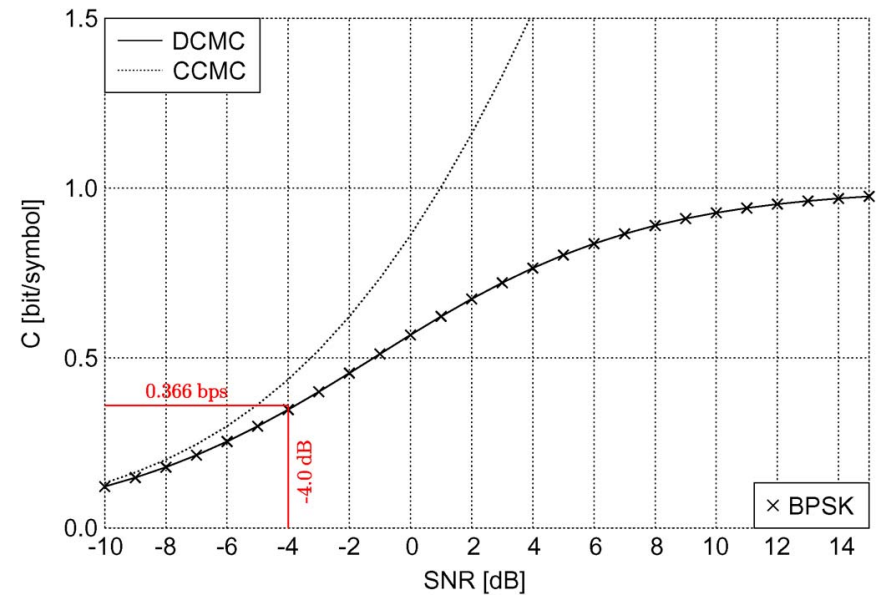

Fig. 5. DCMC and CCMC capacity curves, where the curves were computed based on [24].

cates the requirement of a minimum SNR of $\Gamma_{\lim }=-4 \mathrm{~dB}, 328$ as shown in Fig. 5. Note that $\Gamma_{\text {lim }}$ is represented with the aid of 329 vertical lines in Fig. 4.

As expected, the proposed scheme benefits from the side 331 information constituted by $\left\{b_{2}\right\}$ while compressing the source 332 sequence $\left\{b_{1}\right\}$. Note in Fig. 4 that all DSC schemes outperform 333 the conventional TTCM-QPSK benchmark scheme dispensing 334 with joint decoding, which is labeled by the diamond markers, 335 regardless of the correlation $\rho$, except for the very low correla- 336 tion scenario ${ }^{5}$ of $\rho=0.4$. More explicitly, at a BER $=10^{-5}, 337$ the proposed DSC has an SNR gain of 7.3, 6.2, and $3 \mathrm{~dB} 338$ for $\rho=0.86, \rho=0.8$, and $\rho=0.6$, respectively. However, as 339 expected, with a low correlation value, the proposed scheme 340 has an SNR loss of $1.3 \mathrm{~dB}$, namely, when we have $\rho=0.4$. 341 Again, this is not unexpected because, for $\rho<0.5$, the sources 342 may be deemed to be uncorrelated; hence, they in fact provide 343 misinformation misleading the joint decoder. It may be readily 344 observed in Fig. 4 that, at BER $=10^{-5}$, the scheme having $\rho=345$ 0.86 has the minimum distance with respect to the SW/S limit, 346 i.e., we have $\Gamma-\Gamma_{\lim }=(-3.4)-(-4)=0.6 \mathrm{~dB}$, whereas the 347 scheme associated with $\rho=0.4$ has a distance of $1.7 \mathrm{~dB}$ from 348 the limit.

The effect of the number of iterations between the TCM 350 decoders in Fig. $3 I$, on the overall DSTTCM-QPSK/BPSK 351 scheme's performance, is shown in Fig. 6. It can be observed 352 that doubling the number of iterations from $I=2$ to $I=4353$ will improve the scheme's performance by $1.5 \mathrm{~dB}$, whereas 354 doubling the complexity further will only enhance the system's 355 performance by $0.5 \mathrm{~dB}$. However, doubling the complexity 356 beyond $I=8$ would not provide any further gain at the cost 357 of increasing the decoding complexity; hence, we invoke eight 358 iterations in our decoder.

The SW theoretical bound and the achievable rates obtained 360 for the proposed DSTTCM-QPSK/BPSK schemes are shown, 361 respectively, in Fig. 7. The rates achieved correspond to a 362 BER of $10^{-5}$, and on average, the system's throughput is only 363 0.088 bits away from the bound shown in Fig. 4. Table I 364 summarized the performance of the proposed scheme.

365

\footnotetext{
${ }^{5}$ The higher the crossover probability $p_{e}$, the lower the correlation between the two sources.
} 


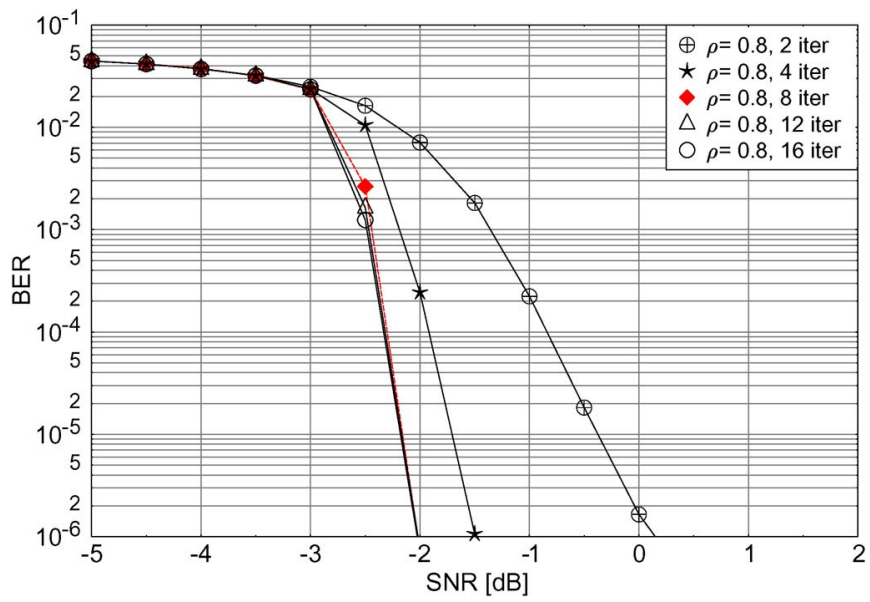

Fig. 6. BER versus SNR performance of the proposed DSTTCMQPSK/BPSK scheme for the correlation parameter of $\rho=\{0.8\}$ when transmitting over uncorrelated Rayleigh fading channels. The number of decoding iterations between the two TCM MAP decoders in Fig. 3 are $I=$ $\{2,4,8,12,16\}$.

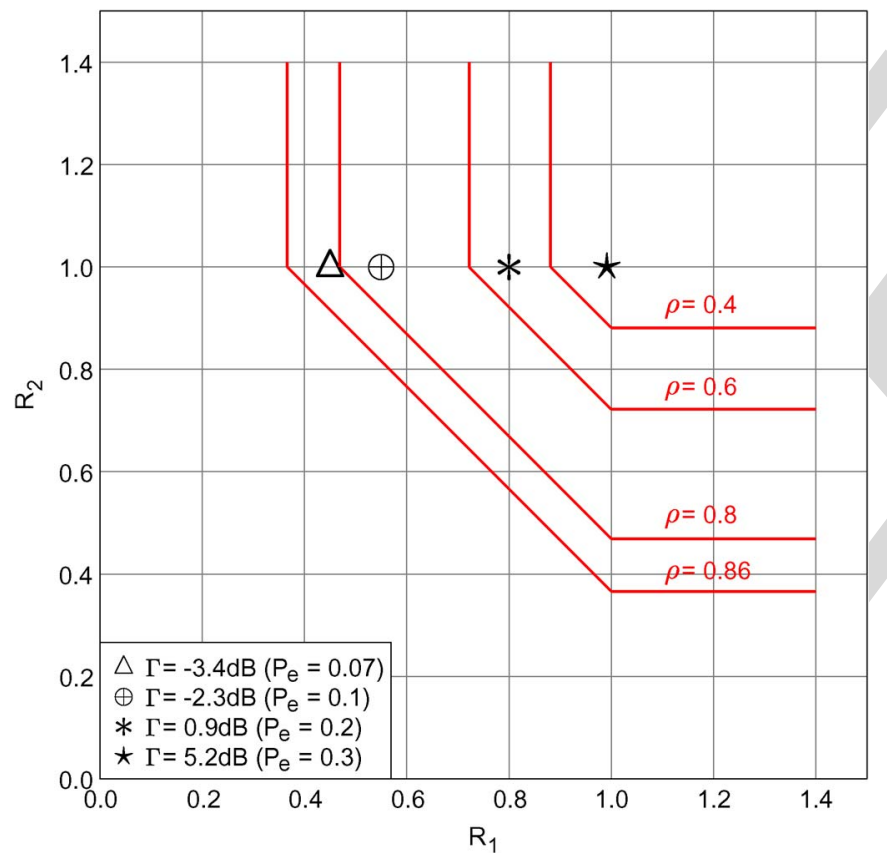

Fig. 7. Theoretical $\mathrm{SW}$ bound and the rates $\left(R_{1}, R_{2}\right)$ achieved by the proposed DSTTCM-QPSK/BPSK scheme for different $p_{e}$ values, where $\Gamma$ denotes the SNR required for achieving a BER $=10^{-5}$.

TABLE I

SySTEM PERFOMANCE OF THE DSTTCM FOR SW CODING AT BER $=10^{-5}$

\begin{tabular}{|c|c|c|c|c|c|c|c|}
\hline$p_{e}$ & $\rho$ & $\eta_{S W}$ & $\eta_{10^{-5}}$ & SW-Gap (bits) & $\Gamma_{\lim }$ & $\Gamma$ & Gap (dB) \\
\hline 0.07 & 0.86 & 0.366 & 0.41 & 0.084 & -4.0 & -3.4 & 0.6 \\
\hline 0.1 & 0.80 & 0.469 & 0.55 & 0.081 & -3.1 & -2.3 & 0.80 \\
\hline 0.2 & 0.60 & 0.722 & 0.8 & 0.078 & 0 & 0.9 & 0.90 \\
\hline 0.3 & 0.40 & 0.881 & 1.0 & 0.108 & 3.5 & 5.2 & 1.7 \\
\hline
\end{tabular}

\section{Simulation Results}

367 Our proposed design has also been extended to higher order 368 modulation modes to conceive an adaptive scheme. Fig. 8 369 shows the BER performance of the different DSTTCM modes 370 for $\rho=0.9$ and $\rho=0.8$ associated with crossover probabilities

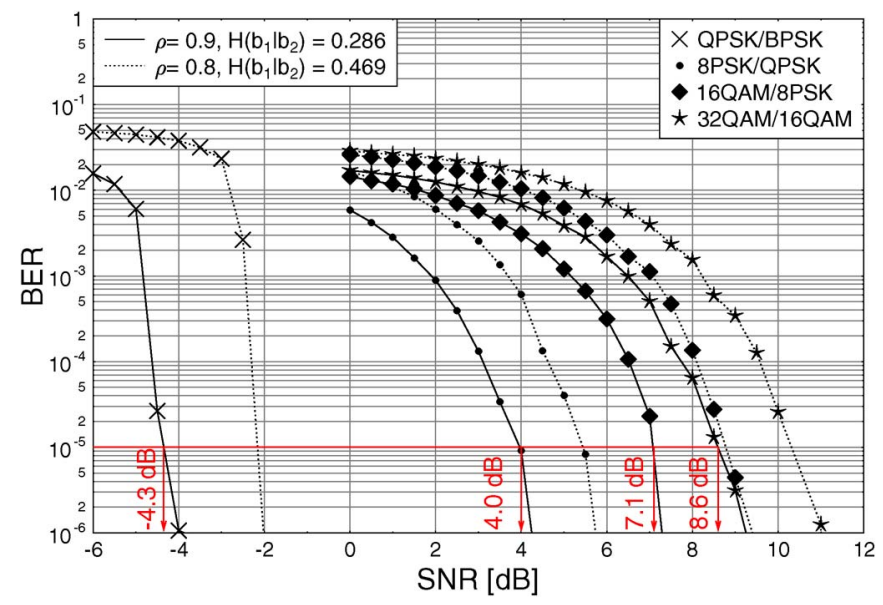

Fig. 8. BER versus SNR performance of the different TTCM modes, when using a block length of $N_{S}=12000$ symbols for $\rho=0.9$ and $\rho=0.86$ for transmission over uncorrelated Rayleigh fading channels.

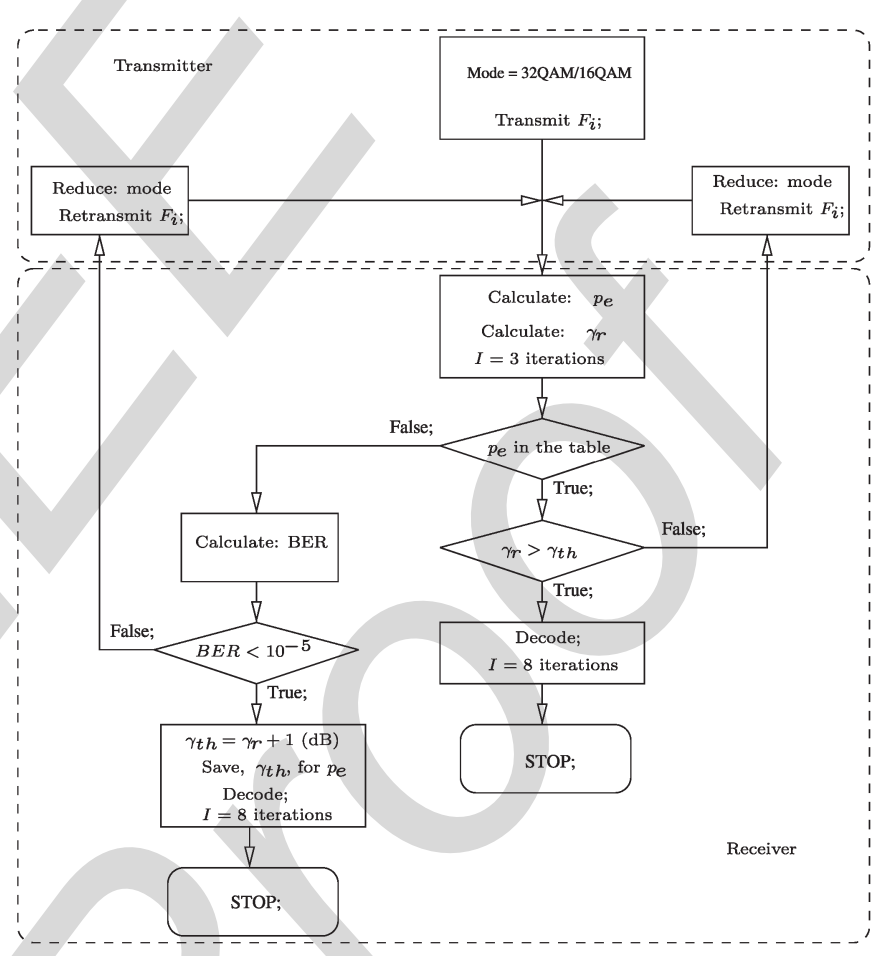

Fig. 9. Flowchart of the adaptive scheme.

of $p_{e}=0.05$ and $p_{e}=0.1$, respectively, while using a block 371 length of $N_{S}=12000$ symbols for all the modulation modes. 372 A total of 10000 blocks have been used in our simulations. 373 The performance of the higher order modulation schemes 374 shown in Fig. 8 suggests that the A-DSTTCM is readily ap- 375 plicable to SW coding. In each mode, we puncture the least 376 significant bit of each coded symbol, which results in punc- 377 turing rates of $R_{p}=\{2 / 1,3 / 2,4 / 3,5 / 4\}$ for QPSK/BPSK, 378 8PSK/QPSK, 16QAM/8PSK, and 32QAM/16QAM, respec- 379 tively. By comparing Figs. 4 and 8, observe that, as expected, 380 the "QPSK/BPSK" scheme outperforms its counterparts in 381 terms of its BER performance since it has a lower throughput. 382

The flowchart in Fig. 9 shows the adaptation process, where 383 the current frame $F_{i}$ will be transmitted first by the highest 384 order 32QAM/16QAM modulation mode. Then, the crossover 385 


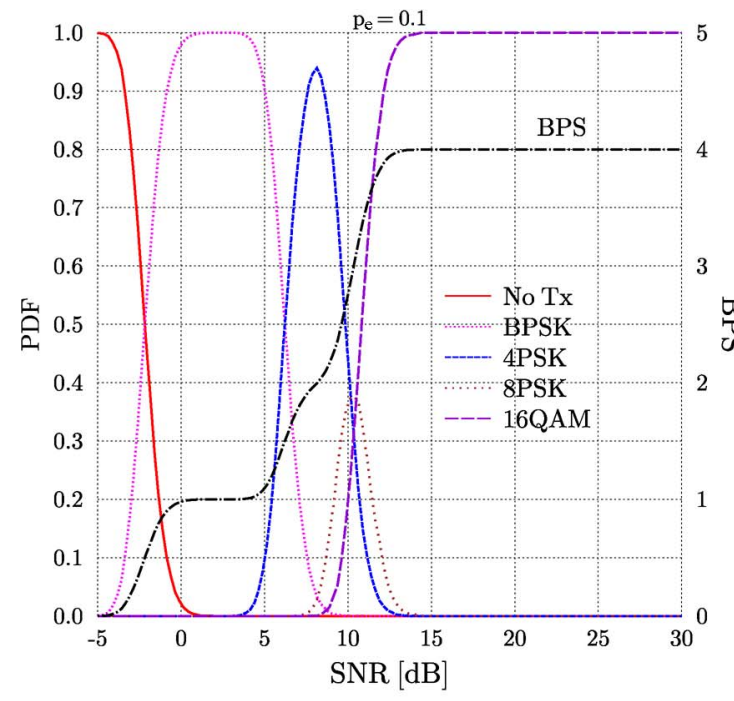

(a)

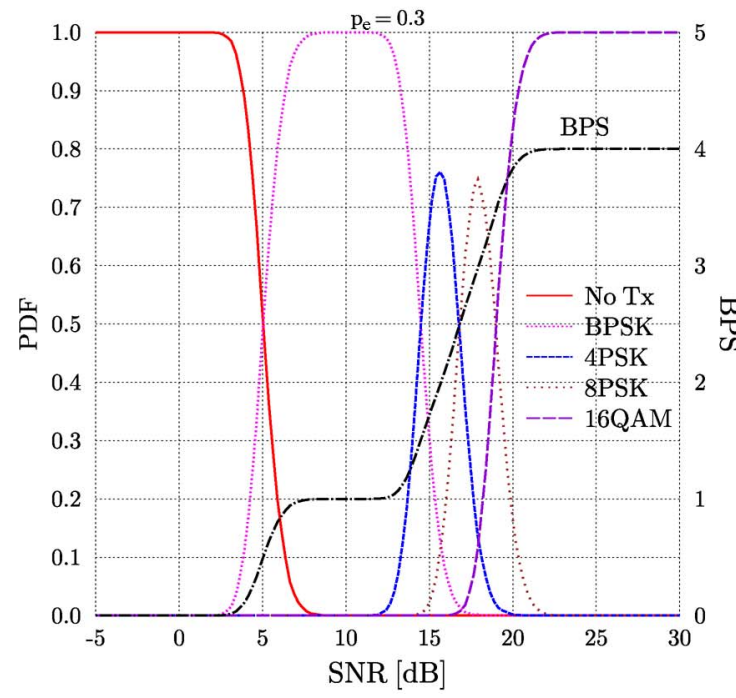

(c)

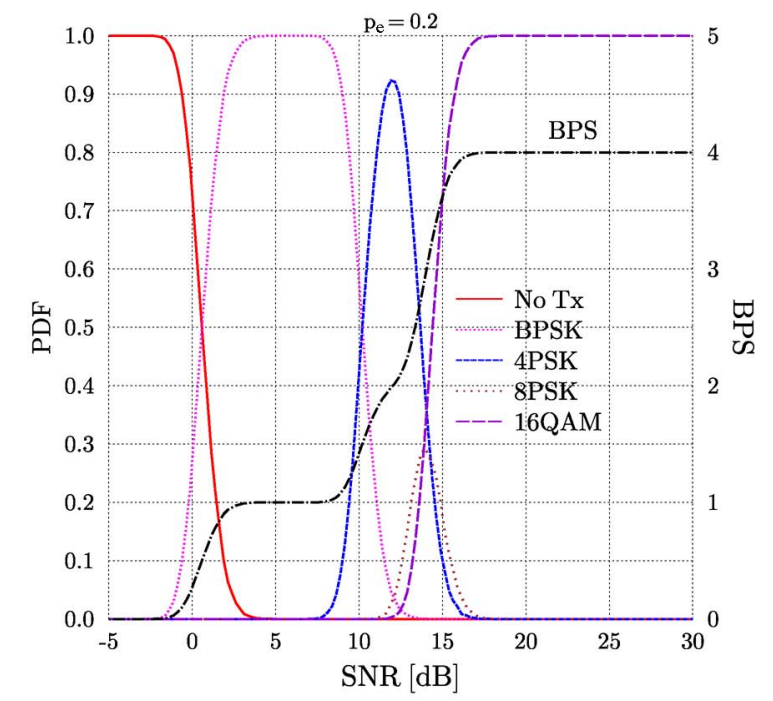

(b)

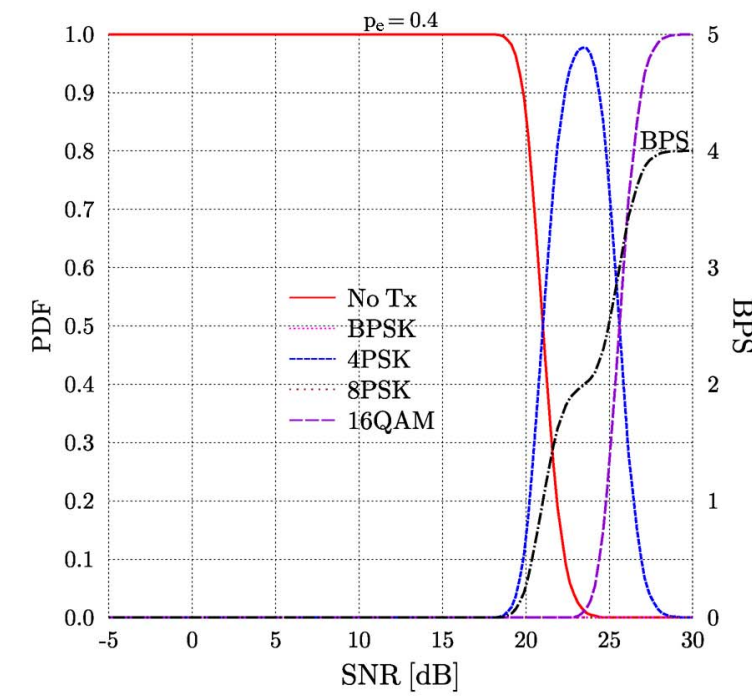

(d)

Fig. 10. PDF of all DSTTCM modes (left-hand vertical axis) versus the received SNR values and the corresponding BPS (right-hand vertical axis) versus the received SNR for $\rho=\{0.8,0.6,0.4,0.2\}$ when communicating over a Rayleigh fading channel. (a) $\rho=0.8$. (b) $\rho=0.6$. (c) $\rho=0.4$. (d) $\rho=0.2$.

386 probability $p_{e}$, which controls the correlation parameter of $\rho=$ $3871-2 p_{e}$, is estimated as [13]

$$
\begin{aligned}
\hat{p}_{e}=\frac{1}{N} \sum_{k=1}^{N} \operatorname{Pr}\left(b_{1}=+1\right) & \operatorname{Pr}\left(b_{2}=-1\right) \\
& +\operatorname{Pr}\left(b_{1}=-1\right) \operatorname{Pr}\left(b_{2}=+1\right) .
\end{aligned}
$$

388 As few as three iterations are sufficient for estimating the 389 correlation parameter sufficiently accurately. Next, the near390 instantaneous SNR $\gamma_{r}$ is calculated using the following:

$$
\gamma_{r}=10 \log _{10}\left(|h|^{2} / N_{0}\right)[\mathrm{dB}] \text {. }
$$

391 Then, the decoder will compare the estimated probability $\hat{p}_{e}$ to 392 the prestored lookup table. The aim of this table is to reduce 393 the overall complexity of the adaptation process. As shown 394 in the flowchart, if $p_{e}$ is not found in the table, the decoder 395 will estimate the BER of the received block, where the BER is 396 estimated after decoding by comparing the source sequence bits $\left\{b_{1}\right\}$ with the decoded bits $\left\{\hat{b}_{1}\right\}$. Provided that the estimated 397 BER is below $10^{-5}$, the corresponding SNR $\gamma_{r}$, which are 398 indicated by the vertical arrows in Fig. 8 will be saved in the 399 lookup table as the threshold of $\gamma_{\mathrm{th}}=\gamma_{r}+1(\mathrm{~dB})$. Note that 400 the additional $1 \mathrm{~dB}$ and the three iterations used will reassure 401 that the performance of the adaptive scheme will remain below 402 a BER of $10^{-5}$. However, when we have BER $<10^{-5}$ or $\gamma_{r}<403$ $\gamma_{\text {th }}$, a feedback acknowledgement will be transmitted to the 404 transmitter that triggers a reduction of the transmission mode 405 index/throughput. We opt to start with a higher rate mode to 406 reduce the number of feedback requests. ${ }^{6}$ Hence, the overall 407 complexity and time required for performing this adaptive rate 408 adjustment process has been reduced.

Furthermore, the probability density function (pdf) of each 410 DSTTCM mode versus the average SNR is shown in Fig. 10, 411 along with the related BPS curves recorded for different 412

\footnotetext{
${ }^{6}$ An incremental mode-based adaptive scheme can be also implemented, subject to additional considerations.
} 
413 correlation of $\rho=\{0.8,0.6,0.4,0.2\}$, respectively. Naturally, 414 the choice of the specific DSTTCM modes is governed by 415 the particular near-instantaneous SNR experienced by the in416 dividual transmission frames at any specific average SNR value 417 in Fig. 10. The adaptive scheme maintains BER $<10^{-5}$. It is 418 shown in Fig. 10 that, as the SNR increases, the higher order 419 DSTTCM modes are activated more often than the lower rate 420 ones. Consequently, the effective BPS throughput increases 421 smoothly with the SNR. It may be also observed from the figure 422 that, in the presence of a high correlation between the two 423 sources, such as $\rho=0.8$, the no transmission mode has effec424 tively disappeared. However, for low correlations, the scheme 425 requires a higher SNR for achieving a nonzero throughput. The 426 adaptive procedure operated as follows. First, the BER of each 427 received block is estimated. If we have BER $<10^{-5}$, then a 428 higher order modulation mode will be requested for the next 429 block.

\section{CONCLUSION}

431 In this paper, we have proposed a novel DSTTCM scheme for 432 a SW-distributed coding system. The proposed system outper433 formed the benchmark scheme dispensing with joint decoding 434 for a wide range of crossover probabilities. A modified symbol435 based MAP was proposed for exploiting the side information 436 available. The theoretical SW/S bounds were estimated, and 437 the proposed scheme was shown to operate within $0.7 \mathrm{~dB}$ 438 from it, for $\rho=0.86$ at a BER of $10^{-5}$. Our proposed scheme 439 operates within 0.088 bits of the maximum achievable rate 440 limit on average, which matches the best result reported in 441 the literature for similar systems while considering a realistic 442 Rayleigh fading channel model. Furthermore, a bandwidth443 efficient practical adaptive scheme was proposed based on a 444 range of adaptive modem modes for transmitting correlated 445 signals over uncorrelated Rayleigh fading channels. The trans446 mitter adapts its coding and modulation modes according to 447 both the short-term channel conditions and to the crossover 448 probabilities for ensuring that the BER remains below $10^{-5}$.

\section{REFERENCES}

[9] J. Garcia-Frias, “Compression of correlated binary sources using turbo 472 codes," IEEE Commun. Lett., vol. 5, no. 10, pp. 417-419, Oct. 2001.473

[10] D. Varodayan, A. Aaron, and B. Girod, "Rate-adaptive codes for dis- 474 tributed source coding," Elsevier Signal Process., vol. 86, no. 11, 475 pp. 3123-3130, Nov. 2006.

[11] A. Liveris, Z. Xiong, and C. Georghiades, "Compression of binary sources 477 with side information at the decoder using LDPC codes," IEEE Commun. 478 Lett., vol. 6, no. 10, pp. 440-442, Oct. 2002.

[12] M. Sartipi and F. Fekri, "Distributed source coding using short to mod- 480 erate length rate-compatible LDPC codes: The entire slepian-wolf rate 481 region," IEEE Trans. Commun., vol. 56, no. 3, pp. 400-411, Mar. 2008.482

[13] J. Garcia-Frias and Y. Zhao, "Near-Shannon/Slepian-Wolf performance 483 for unknown correlated sources over AWGN channels," IEEE Trans. 484 Commun., vol. 53, no. 4, pp. 555-559, Apr. 2005.

[14] Y. Zhao, W. Zhong, and J. Garcia-Frias, "Transmission of correlated 486 senders over a Rayleigh fading multiple access channel," Elsevier Signal 487 Process., vol. 86, no. 11, pp. 3150-3159, Apr. 2006.

[15] J. Del Ser, P. Crespo, and A. Munoz, "Joint source-channel decoding 489 of correlated sources over ISI channels," in Proc. IEEE VTC Spring, 490 Jun. 2005, vol. 1, pp. 625-629.

[16] K. Anwar and T. Matsumoto, "Iterative spatial demapping for two corre- 492 lated sources with power control over fading MAC," in Proc. IEEE VTC 493 Spring, May 2012, pp. 1-7.

[17] L. Hanzo, T. H. Liew, B. L. Yeap, and S. X. Ng, Turbo Coding, Turbo 495 Equalisation and Space-Time Coding: EXIT-Chart Aided Near-Capacity 496 Designs for Wireless Channels. Hoboken, NJ, USA: Wiley, 2010. 497

[18] D. Varodayan, Y.-C. Lin, and B. Girod, "Adaptive distributed source 498 coding," IEEE Trans. Image Process., vol. 21, no. 5, pp. 2630-2640, 499 May 2012.

[19] A. Roumy, K. Lajnef, and C. Guillemot, "Rate-adaptive turbo- 501 syndrome scheme for slepian-wolf coding," in Proc. ACSSC, Nov. 2007, 502 pp. 545-549.

[20] X. Lv, R. Liu, and R. Wang, "A novel rate-adaptive distributed source 504 coding scheme using polar codes," IEEE Commun. Lett., vol. 17, no. 1, 505 pp. 143-146, Jan. 2013.

[21] L. Cui, S. Wang, S. Cheng, and M. Yeary, "Adaptive binary slepian-wolf 507 decoding using particle based belief propagation," IEEE Trans. Commun., 508 vol. 59, no. 9, pp. 2337-2342, Sep. 2011.

509

[22] P. Robertson and T. Wörz, "Bandwidth-efficient turbo trellis-coded mod- 510 ulation using punctured component codes," IEEE J. Sel. Areas Commun., 511 vol. 16, no. 2 , pp. 206-218, Feb. 1998.

[23] V. Toto-Zarasoa, A. Roumy, and C. Guillemot, "Rate-adaptive codes for 513 the entire slepian-wolf region and arbitrarily correlated sources," in Proc. 514 IEEE ICASSP, Apr. 2008, pp. 2965-2968.

[24] S. X. Ng and L. Hanzo, "On the MIMO channel capacity of multi- 516 dimensional signal sets," IEEE Trans. Veh. Technol., vol. 55, no. 2, 517 pp. 528-536, Mar. 2006.

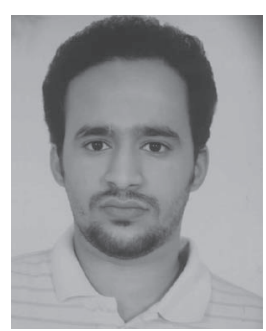

Abdulah Jeza Aljohani received the B.S. degree 519 (with honors) in electronics and communication en- 520 gineering from King Abdulaziz University, Jeddah, 521 Saudi Arabia, in 2006 and the M.Sc. degree (with 522 distinction) in wireless communications from the 523 University of Southampton, Southampton, U.K., in 524 2009. He is currently working toward the Ph.D. 525 degree with the Communications, Signal Processing, 526 and Control Group, School of Electronics and Com- 527 puter Science, University of Southampton. 528

His current research interests include joint source/ 529 channel coding and distributed source coding. 


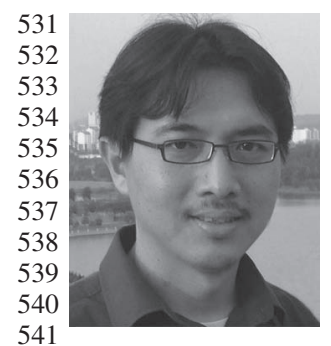

Soon Xin Ng (S'99-M'03-SM'08) received the B.Eng. degree (with first-class honors) in electronics engineering and the Ph.D. degree in wireless communications from the University of Southampton, Southampton, U.K., in 1999 and 2002, respectively.

From 2003 to 2006, he was a Postdoctoral Research Fellow working on collaborative European research projects known as SCOUT, NEWCOM, and PHOENIX. Since August 2006, he has been with the School of Electronics and Computer Science, University of Southampton, where he is currently a 542 Senior Lecturer. He is involved in the OPTIMIX and CONCERTO European 543 projects, as well as the IU-ATC and UC4G projects. He is the author of over 544150 papers and the coauthor of two John Wiley/IEEE Press books. His research 545 interests include adaptive coded modulation, coded modulation, channel 546 coding, space-time coding, joint source and channel coding, iterative detection, 547 orthogonal frequency-division multiplexing, multiple-input-multiple-output 548 systems, cooperative communications, distributed coding, quantum error549 correcting codes, and joint wireless-and-optical-fiber communications.

550 Dr. $\mathrm{Ng}$ is a Chartered Engineer and a Fellow of the Higher Education 551 Academy in the U.K.

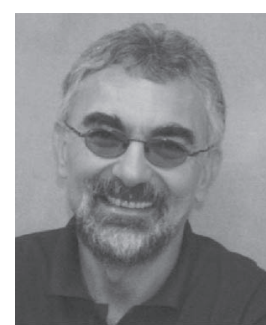

Lajos Hanzo (F'08) received the M.S. degree in 552 electronics, the D.Sc. degree, and the Doctor Honoris 553 Causa degree from the Technical University of Bu- 554 dapest, Budapest, Hungary, in 1976, 1983, and 2009, 555 respectively.

556

During his 37-year career in telecommunications, 557 he has held various research and academic posts in 558 Hungary, Germany, and the U.K. From 2008 to 2012, 559 he was a Chaired Professor with Tsinghua Univer- 560 sity, Beijing, China. Since 1986, he has been with 561 the School of Electronics and Computer Science, 562 University of Southampton, Southampton, U.K., where he is currently the Chair 563 of telecommunications. He is the coauthor of 20 John Wiley/IEEE Press books 564 on mobile radio communications, totalling in excess of 10000 pages, and the 565 author of more than 1300 research entries at IEEE Xplore. He has successfully 566 supervised more than $80 \mathrm{Ph} . \mathrm{D}$. students. He is an enthusiastic supporter of 567 industrial and academic liaison, and he offers a range of industrial courses. 568 Currently, he is directing a 100-strong academic research team, working on a 569 range of research projects in the field of wireless multimedia communications 570 sponsored by industry, the Engineering and Physical Sciences Research Council 571 U.K., the European Research Council's Advanced Fellow Grant, and the 572 Royal Society's Wolfson Research Merit Award. His research is funded by 573 the European Research Council's Senior Research Fellow Grant. For further 574 information on research in progress and associated publications, see (http:// 575 www-mobile.ecs.soton.ac.uk). He has $17000+$ citations.

Dr. Hanzo is a Fellow of the Royal Academy of Engineering, the Institution 577 of Engineering and Technology, and the European Association for Signal 578 Processing. He has served both as a Technical Program Committee and General 579 Chair of IEEE conferences, has presented keynote lectures, and has been 580 awarded a number of distinctions. He is also a Governor of the IEEE Vehicular 581 Technology Society. 
AUTHOR QUERY

NO QUERY.

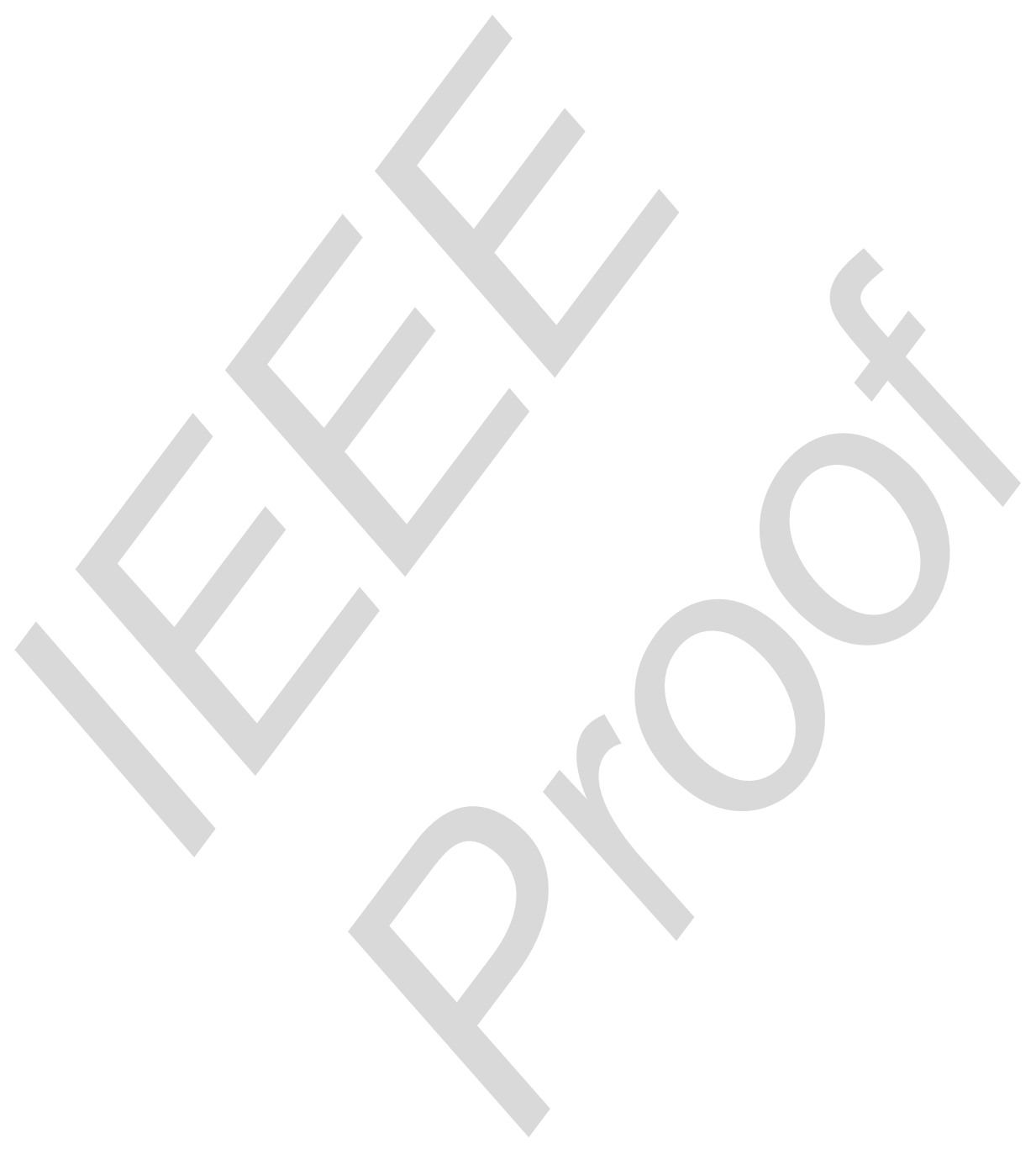




\title{
TTCM-Aided Rate-Adaptive Distributed Source Coding for Rayleigh Fading Channels
}

\author{
Abdulah Jeza Aljohani, Soon Xin Ng, Senior Member, IEEE, and Lajos Hanzo, Fellow, IEEE
}

\begin{abstract}
4 Abstract-Adaptive turbo-trellis-coded modulation (TTCM)5 aided asymmetric distributed source coding (DSC) is proposed, 6 where two correlated sources are transmitted to a destination 7 node. The first source sequence is TTCM encoded and is further 8 compressed before it is transmitted through a Rayleigh fading 9 channel, whereas the second source signal is assumed to be per10 fectly decoded and, hence, to be flawlessly shown at the des11 tination for exploitation as side information for improving the 12 decoding performance of the first source. The proposed scheme 13 is capable of reliable communications within $0.80 \mathrm{~dB}$ of the 14 Slepian-Wolf/Shannon (SW/S) theoretical limit at a bit error rate 15 (BER) of $\mathbf{1 0}^{-5}$. Furthermore, its encoder is capable of accom16 modating time-variant short-term correlation between the two 17 sources.
\end{abstract}

18 Index Terms-Distributed source coding (DSC), joint source19 channel coding, Slepian-Wolf (SW) coding, Turbo-trellis-oded 20 modulation (TTCM).

\section{INTRODUCTION}

${ }^{2} \mathbf{D}$ ISTRIBUTED source coding (DSC) [1] refers to the problem of compressing several physically separated, but cor24 related sources, where the receiver can perform joint decoding 25 of both encoded signals. The schematic of asymmetric DSC [2] 26 is shown in Fig. 1, where source sequence $\left\{b_{1}\right\}$ is compressed 27 before its transmission, whereas the correlated source signal $28\left\{b_{2}\right\}$ is assumed to be available at the decoder but not at the 29 source $\left\{b_{1}\right\}$. More explicitly, the encoder has to compress $\left\{b_{1}\right\}$ 30 without knowing $\left\{b_{2}\right\}$, yet the decoder is capable of exploiting 31 the knowledge of $\left\{b_{2}\right\}$ for recovering $\left\{b_{1}\right\}$.

32 More explicitly, the Slepian-Wolf (SW) [3] coding theorem 33 specifies the achievable rate regions of the compressed corre34 lated sources $\left\{b_{1}\right\}$ and $\left\{b_{2}\right\}$ for transmission to a joint decoder 35 as $R_{1} \geq H\left(b_{1} \mid b_{2}\right), R_{2} \geq H\left(b_{2} \mid b_{1}\right)$, and $R_{1}+R_{2} \geq H\left(b_{1}, b_{2}\right)$, 36 where $H\left(b_{1} \mid b_{2}\right)$ and $H\left(b_{1}, b_{2}\right)$ denote the conditional entropy 37 and joint entropy, respectively. Remarkably, this bound is 38 identical, regardless whether joint encoding or joint decoding 39 is used, i.e., regardless of where the joint processing takes

Manuscript received July 8, 2013; revised September 30, 2013; accepted October 1, 2013. This work was supported in part by the Ministry of Higher Education of Saudi Arabia, by the European Union's Seventh Framework Programme through the CONCERTO Project under Grant 288502, by the Research Councils U.K. through the India-U.K. Advanced Technology Centre, by the European Research Council under an Advanced Fellow Grant, and by the Royal Society under the Wolfson Research Merit Award. The review of this paper was coordinated by Dr. E. K. S. Au.

The authors are with the Communications, Signal Processing, and Control Research Group, School of Electronics and Computer Science, University of Southampton, Southampton SO17 1BJ, U.K. (e-mail: ajra1c09@ecs.soton. ac.uk; sxn@ecs.soton.ac.uk; 1h@ecs.soton.ac.uk).

Color versions of one or more of the figures in this paper are available online at http://ieeexplore.ieee.org.

Digital Object Identifier 10.1109/TVT.2013.2285020

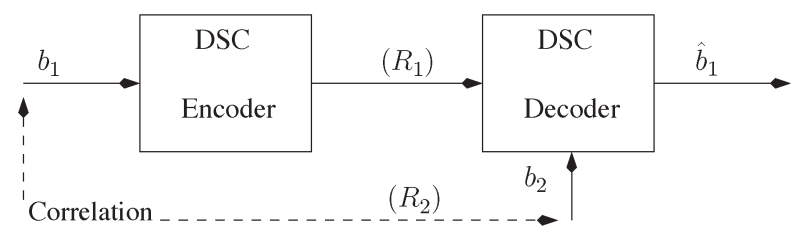

Fig. 1. Schematic of the asymmetric DSC.

place. This is quite convenient for exploiting the correlation of 40 sources, which are distant from each other with the aid of joint 41 processing at the receiver. This would facilitate for example 42 the efficient joint decoding of correlated camera-phone-video 43 sequences at the base station, namely, sequences, which portray 44 the same scene from different angles.

This promising theoretical result has led to an increasing 46 interest in a variety of applications, such as sensor networks 47 [1], robust wireless video transmission [4], and compression 48 of secure biometric data [5], where exchanging information 49 between source nodes is not possible or not practical. Applying 50 DSC techniques in wireless sensor networks, for example, 51 has led to a new processing paradigm, where the potential 52 computational complexity has been moved from the battery- 53 limited sources to the central decoder connected to the mains 54 supply.

As a consequence, the critical power constraint, which di- 56 rectly predetermines the life span of the wireless node, is 57 fulfilled [1]. The first practical DSC technique was proposed 58 in [6], where both sources $\left\{b_{1}\right\}$ and $\left\{b_{2}\right\}$ are assumed to be 59 emitting equiprobable codewords, but they exhibit a difference 60 because $\left\{b_{2}\right\}$ is known only to the joint decoder but not to the 61 encoder of $\left\{b_{1}\right\}$. Naturally, this assumption does not preclude 62 that the codewords of $\left\{b_{2}\right\}$ actually received from a remote 63 source, but they must be first perfectly recovered in isolation 64 before they may be used by the joint decoder for recovering 65 $\left\{b_{1}\right\}$. Then, the codewords of both sources are grouped in 66 cosets, where the members of each coset are separated by 67 the maximum possible Hamming distance. Given $\left\{b_{2}\right\}$ at the 68 receiver, it is sufficient to transmit the index of the specific coset 69 hosting the codewords of $\left\{b_{1}\right\}$. The decoder then estimates the 70 transmitted codeword by choosing the one that is closest to the 71 side information constituted by $\left\{b_{2}\right\}$ of a given coset in terms 72 of the Hamming distance.

The idea of using channel coding techniques ${ }^{1}$ has enabled 74 practical solutions to be developed. Practical Slepian-Wolf 75

\footnotetext{
${ }^{1}$ Since the correlation between the sources may be interpreted as the ameliorating effect of a "virtual" channel, a good channel code having, for example, a maximum minimum Hamming distance will be a good SW code [7].
} 
76 schemes using turbo codes (TCs) were proposed for example 77 in [2], [8], and [9], whereas low-density parity-check (LDPC) 78 codes were considered in [10]-[12]. However, finding the best 79 code for approaching the Slepian-Wolf/Shannon (SW/S) limit 80 was not considered in [2], [8], and [9]. Later, a so-called "super" $81 \mathrm{TC}$ was proposed in [13], aiming for approaching the SW/S 82 limit, when communicating over additive white Gaussian noise 83 (AWGN) channels. However, the scheme proposed in [13] for 84 an AWGN channel suffers from an error floor when communi85 cating over Rayleigh fading channels that makes the system less 86 suitable for wireless applications. A modified LDPC code was 87 proposed in [14] for mitigating the error floor, but nonetheless, 88 a high error floor persists when the correlation between the 89 sources is low. A joint turbo equalizer and decoder scheme 90 was proposed for asymmetric DSC in [15], whereas an iterative 91 joint turbo equalizer and decoder scheme was conceived for 92 transmission over a multipath Rayleigh fading multiple-access 93 channel in [16]. Both schemes have achieved a near-SW/S 94 performance, albeit at high joint decoding complexity. More 95 specifically, 35 iterations were invoked between the decoder 96 components in [15], whereas as many as 350 iterations were 97 required in [16] for attaining a near-SW/S performance. By 98 contrast, we only invoke eight turbo iterations in our TTCM 99 decoder, where both constituent decoders have comparable 100 complexity [17].

101 Furthermore, in practice, the short-term correlation among 102 the sources might be time variant; hence, adaptive-rate schemes 103 have to be considered, where the code rate is controlled via 104 a feedback channel. More specifically, if the bit error rate 105 (BER) evaluated after decoding exceeds a given threshold, 106 more syndromes (or parity bits if parity puncturing is used) 107 will be requested from the transmitter. A pair of innovative 108 adaptive-rate LDPC schemes was proposed in [18], whereas 109 adaptive-rate TCs were designed in [19]. In [18], the encoder 110 stored the syndromes and incrementally transmitted them to 111 the receiver, when the decoder failed to find the legitimate 112 codeword. Both papers considered an asymmetric DSC struc113 ture based on the puncturing of the syndrome generated by the 114 channel encoders, while stipulating the idealized simplifying 115 assumption of modeling the channel as the parallel combination 116 of a perfect channel and a binary symmetric channel (BSC). 117 More advanced adaptive-rate schemes considered the employ118 ment of a polar code [20] or efficient particle-based belief119 propagation-aided decoding [21] and density-evolution-based 120 decoding techniques [18].

121 Against this background, we propose a novel bandwidth122 efficient turbo-trellis-coded modulation (TTCM) scheme, 123 which combines the functions of coding and modulation for 124 conceiving a new DSCs system. TTCM [22] has a structure 125 similar to that of the family of binary TCs, where two identical 126 parallel-concatenated TCM schemes rather than conventional 127 codes are employed as component codes. The classic TTCM 128 design was outlined in [22], which is based on the search for 129 the best TCM component codes using the so-called "punctured" 130 minimal distance criterion, to approach the capacity of the 131 AWGN channel. The TTCM code advocated was designed to 132 improve the attainable throughput by considering the design of 133 error-correcting code and modulation where the parity bits are absorbed in without any bandwidth expansion by increasing 134 the number of bits per modulated symbol. By contrast, all 135 separated channel codes, such as turbo or LDPC codes, impose 136 a bandwidth expansion, which is proportional to the code rate. 137 Furthermore, a novel adaptive-rate mechanism is conceived for 138 increasing the system's effective throughput, while ensuring an 139 infinitesimally low BER. Hence, our new contributions are as 140 follows.

- We propose a uniquely amalgamated DSC and TTCM 142 (DSTTCM) scheme for SW coding, which is capable of 143 attaining a near-SW/S performance for a wide range of 144 source correlation values. Our new scheme exhibits no 145 error floor, ${ }^{2}$ despite its low complexity. Furthermore, a 146 carefully constructed modified symbol-based maximum 147 a posteriori (MAP) algorithm is conceived for exploiting 148 the side information available at the decoder. Additionally, 149 we eliminate the aforementioned idealized simplifying 150 assumptions exploited in the prior literature [18], [19], 151 [23], and consider more realistic uncorrelated Rayleigh 152 fading channels and BSCs.

- Furthermore, adaptive DSTTCM (A-DSTTCM) is pro- 154 posed for accommodating the near-instantaneously time- 155 varying nature of the wireless channel and the short-term 156 correlation fluctuations among the sources. More explic- 157 itly, the system adapts its parameters according to both the 158 channel quality and the correlation $\rho$ of the sources, ${ }^{3}$ while 159 maintaining a given target BER.

The remainder of this paper is organized as follows. The 161 proposed system model is described in Section II. The proposed 162 scheme is designed in Section III. Section IV discusses our 163 results. Finally, our conclusions are offered in Section V.

\section{SYSTEM MODEL}

165

The asymmetric DSC scenario in Fig. 1 was considered here, 166 where sequence $\left\{b_{2}\right\}$ is transmitted at the rate of $R_{2}=H\left(b_{2}\right), 167$ which is typically referred to as "side information" in most 168 contributions [2], [8]; again, however, it can be also interpreted 169 as another desired source signal, which was perfectly recovered. 170 Sequence $\left\{b_{2}\right\}$ can be also transmitted through an independent 171 Rayleigh-fading channel, in which case, a similar encoder 172 structure to that of the first source $\left\{b_{1}\right\}$ has to be implemented/ 173 The problem in this scenario may be considered a symmetric 174 DSC one. Symmetric DSC compression was discussed, for 175 example, in [13], [14], and [16], but due to space limitations, 176 it is beyond the scope of this paper.

Furthermore, crossover probability $p_{e}$ used for modeling 178 correlation parameter $\rho$ may be assumed to be the overall proba- 179 bility of error, which is denoted as $p_{e_{1}}$ and $p_{e_{2}}$. More explicitly, 180 the error between the two sources is denoted by $p_{e_{1}}$ and $p_{e_{2}} 181$ which characterizes the transmission error between the second 182 source and the destination. Thus, in this scenario, the overall 183 error obeys $p_{e} \leq p_{e_{1}}+p_{e_{2}}$. The correlated sequence $\left\{b_{1}\right\}$ is 184 then compressed for approaching the Slepian-Wolf bound to a 185

\footnotetext{
${ }^{2}$ As documented in Fig. 4, no error floor is observed at above BER level of $10^{-6}$; hence, our scheme is evidently suitable for wireless applications.

${ }^{3} \rho$ is expressed using a crossover probability $p_{e}$ as $\rho=1-2 p_{e}$.
} 


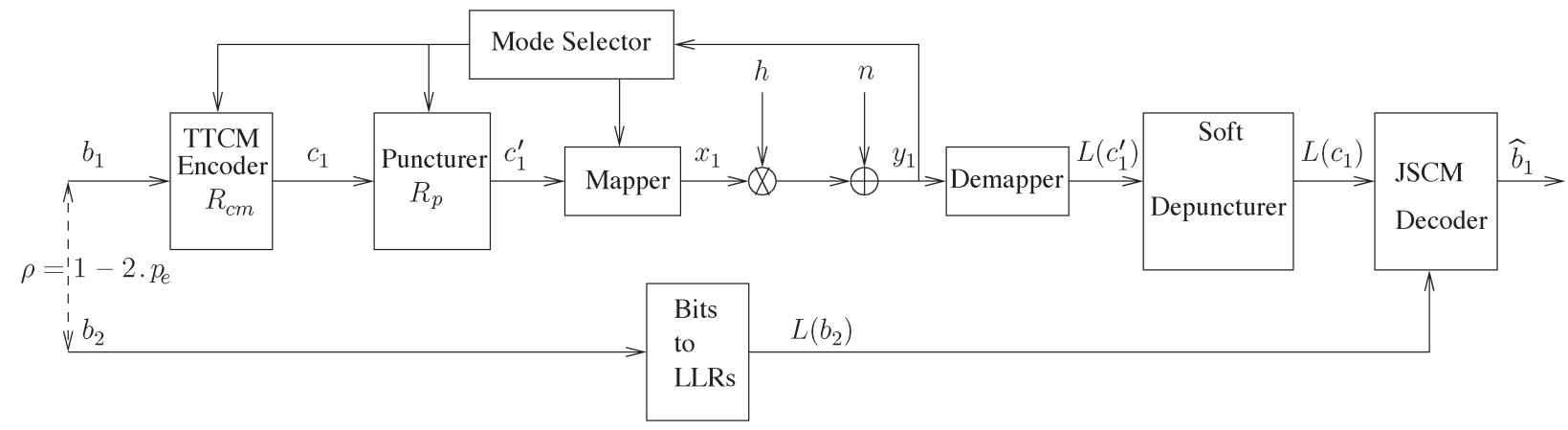

Fig. 2. Block diagram of the A-DSTTCM system communicating over Rayleigh fading channels. Sources $\left\{b_{1}\right\}$ and $\left\{b_{2}\right\}$ are assumed to be correlated, i.e., we have $H\left(b_{1} \mid b_{2}\right) \neq H\left(b_{1}\right)$, and $L(\cdot)$ denotes the LLRs.

186 rate of $R_{1}=H\left(b_{1} \mid b_{2}\right)$ to achieve the overall rate of $H\left(b_{1}, b_{2}\right)$. 187 Typically, the BSC is used for modeling the correlation between 188 the two source sequences $\left\{b_{1}\right\}=\left\{b_{1}^{1}, b_{1}^{2}, \ldots, b_{1}^{i}, \ldots, b_{1}^{N}\right\}$ and $189\left\{b_{2}\right\}=\left\{b_{2}^{1}, b_{2}^{2}, \ldots, b_{2}^{i}, \ldots, b_{2}^{N}\right\}$, where $N$ is the length of each 190 source block.

191 For example, when $\left\{b_{1}\right\}$ and $\left\{b_{2}\right\}$ have a correlation of $192 \rho=0.9$ given the perfect knowledge of $\left\{b_{2}\right\},\left\{b_{1}\right\}$ may be in193 terpreted as the output signal of BSC, which was contaminated 194 by the bit-flipping error events occurring with a probability 195 of $p_{e}$. Source sequence $\left\{b_{1}\right\}$ is generated by an equiprobable 196 binary symmetric independent identically distributed (i.i.d.) 197 source, whereas $\left\{b_{2}\right\}$ can be defined as $b_{1}^{i}=b_{2}^{i} \oplus e_{i}$, where $\oplus$ 198 is the modulo- 2 addition operation, and $e_{i}$ is an independent 199 binary random variable assuming the logical value of 1 with 200 a crossover probability of $p_{e}$ and of 0 with a probability of $201\left(1-p_{e}\right)$. Both the random variables of $b_{1}^{i}$ and $b_{2}^{i}$ in the pair 202 of bit streams $\left\{b_{1}\right\}$ and $\left\{b_{2}\right\}$ may be assumed to be i.i.d. for 203 the bit index $i$; hence, both sources emit equiprobable [16] bits. 204 Consequently, the entropy of each source is unity, which yields 205 conditional entropy of

$$
\begin{aligned}
H\left(p_{e}\right) & =H\left(b_{1} \mid b_{2}\right) \\
& =\lim _{i \rightarrow \infty} \frac{1}{i} H\left[\left(\left(b_{1}^{1}, \cdots, b_{i}^{1}, \cdots b_{N}^{1}\right) \mid\left(b_{1}^{2}, \cdots, b_{i}^{2}, \cdots b_{N}^{2}\right)\right)\right]
\end{aligned}
$$

206 where $H\left(p_{e}\right)=p_{e} \log _{2}\left(1 / p_{e}\right)+\left(1-p_{e}\right) \log _{2}\left(1 / 1-p_{e}\right)$ is 207 the entropy of the binary random variable, and $e_{i}$ is used for 208 parametrizing the side information/ Therefore, the achievable $209 \mathrm{SW}$ rate region is given by the following three inequalities [16]:

$$
\begin{gathered}
R_{1} \geq H\left(p_{e}\right) \\
R_{2} \geq H\left(p_{e}\right) \\
R_{1}+R_{2} \geq 1+H\left(p_{e}\right) .
\end{gathered}
$$

210 Let us now embark on the design of a joint source-channel 211 (JSC) decoding scheme for minimizing the signal-to-noise 212 power ratio (SNR) required for approaching the SW/S bound, 213 while maintaining reliable communications. More explicitly, 214 let us define $\mathrm{SNR}=R_{1} \cdot E_{b} / N_{0}$, where $E_{b} / N_{0}$ denotes the 215 energy per bit to noise power spectral density.

\section{A. Encoder}

The block diagram of the proposed A-DSTTM scheme con- 217 sidered for transmitting correlated sources is shown in Fig. 2, 218 where $L(\cdot)$ denotes the log-likelihood ratios (LLRs) of the bits. 219 As shown in Fig. 2, the input sequence $\left\{b_{1}\right\}$ is fed into a 220 TTCM encoder, which has a coding rate of $R_{\mathrm{cm}}=m / m+1221$ and invokes a $2^{m+1}$-level modulation scheme. The TTCM- 222 encoded bits are then punctured at a rate of $R_{p}$. The resultant bit 223 sequence $\left\{c_{1}^{\prime}\right\}$ is then mapped to the corresponding modulated 224 symbols $\left\{x_{1}\right\}$ before their transmission over an uncorrelated 225 Rayleigh fading channel. The second bit sequence $\left\{b_{2}\right\}$ will 226 be converted to the LLRs $L\left(b_{2}\right)=L_{e}$, which is then will be 227 exploited as side information. This conversion is necessary 228 because the joint decoder is a soft-decision-based one. These 229 LLRs are characterized by the aforementioned crossover prob- 230 ability $p_{e}$ and can be estimated as

$$
\begin{aligned}
L\left(b_{2} \mid b_{1}\right) & =\ln \left[\frac{\operatorname{Pr}\left(b_{2}=+1 \mid b_{1}\right)}{\operatorname{Pr}\left(b_{2}=-1 \mid b_{1}\right)}\right] \\
& =\ln \left[\frac{\left(1-p_{e}\right) \operatorname{Pr}\left(b_{1}=+1\right)+p_{e} \operatorname{Pr}\left(b_{1}=+1\right)}{\left(1-p_{e}\right) \operatorname{Pr}\left(b_{1}=-1\right)+p_{e} \operatorname{Pr}\left(b_{1}=-1\right)}\right] .
\end{aligned}
$$

We assume that these LLRs are available at the destination 232 and to be exploited by the joint decoder, whereas $p_{e}$ can be 233 estimated at the decoder using (11).

As an example, we use a rate $R_{\mathrm{cm}}=1 / 2 \mathrm{TTCM}$ encoder 235 relying on a puncturer of rate $R_{p}=2 / 1$, which punctures 236 one bit out of two encoded bits. We assume that all the 237 systematic bits are punctured, whereas all the parity bits are 238 transmitted to the decoder. Hence, the overall code rate is 239 $R_{1}=R_{\mathrm{cm}} \cdot R_{p}=1$. However, the parity bit sequence may be 240 also further punctured to achieve an increased compression 241 ratio. The resultant bits are then mapped to binary phase-shift 242 keying (BPSK) symbols, i.e., the modulation mode has been 243 changed from quadrature phase-shift keying (QPSK) to BPSK 244 (QPSK/BPSK). Thus, the corresponding effective throughput 245 is given by $\eta=R_{1} \cdot \log _{2}(2)=1$ bits per symbol (BPS). Then, 246 the modulated symbol sequence $\left\{x_{1}\right\}$ is transmitted over an 247 uncorrelated Rayleigh fading channel, and the received symbol 248 $y_{1}$ is given by

$$
y_{1}=h x_{1}+n
$$




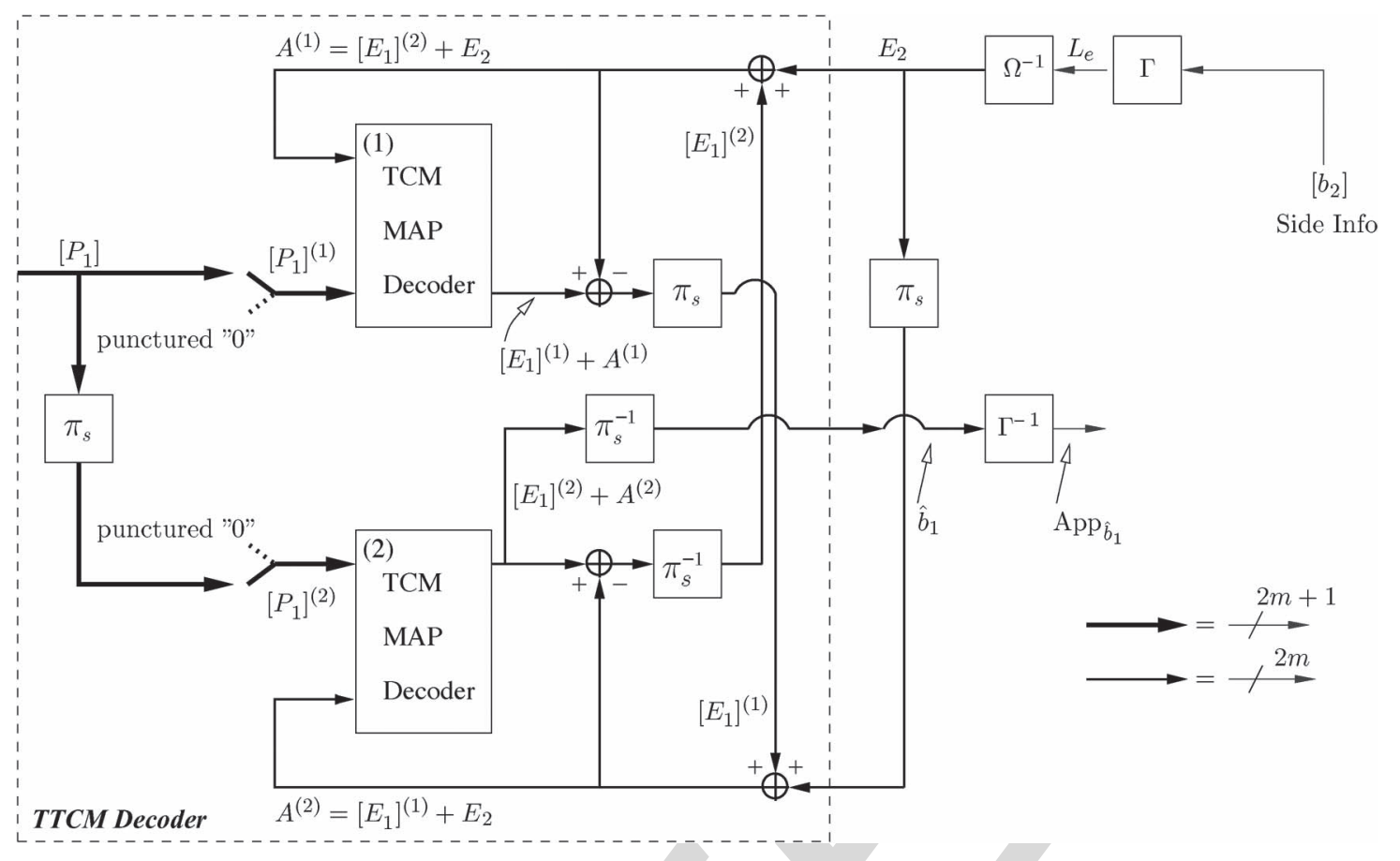

Fig. 3. Block diagram of the JSC-aided TTCM decoder conceived for our DSC system. Notations $\pi_{s}$ and $\pi_{s}^{-1}$ represent the symbol interleaver and deinterleaver, respectively, whereas $\Gamma$ and $\Gamma^{-1}$ denote the relevant symbol-to-LLR and LLR-to-symbol probability conversion.

250 where $h$ is the fading coefficient of the channel, and $n$ is the 251 AWGN having a variance of $N_{0} / 2$ per dimension. The short252 term average received SNR is given by

$$
\mathrm{SNR}_{r}=\frac{E\left\{|h|^{2}\right\} E\left\{\left|x_{1}\right|^{2}\right\}}{N_{0}}
$$

253 where $x_{1}$ represents the modulated symbols of source $\left\{b_{1}\right\}$ 254 after puncturing. Furthermore, we have $E\left\{\left|x_{1}\right|^{2}\right\}=1$, and 255 the SNR of $\gamma_{r}=10 \log _{10}\left(|h|^{2} / N_{0}\right)[\mathrm{dB}]$ is estimated for each 256 received block. Note that $\left\{b_{2}\right\}$ is related to $\left\{b_{1}\right\}$ according 257 to $b_{1}^{i}=b_{2}^{i} \oplus e_{i}$, as detailed in Section II. Diverse effective 258 throughputs may be derived by changing $R_{\mathrm{cm}}$ and $R_{p}$; hence, 259 the proposed scheme exhibits substantial flexibility. In our 260 A-DSTTCM scheme, the following modes are chosen at the 261 encoder to ensure that BER $<10^{-5}$ :

262 - No transmission;

263 - DSTTCM-QPSK/BPSK;

264 - DSTTCM-8PSK/QPSK;

265 - DSTTCM-16-quadrature amplitude modulation (QAM)/ 266 8PSK;

267 - DSTTCM-32QAM/16QAM.

268 Thus, the effective throughput of our adaptive system as269 sumes the values of $\eta=\{0,1,2,3,4\}$ BPS.

\section{B. Joint Source-Channel Decoder}

271 Our JSC-decoding-aided DSTTCM scheme is shown in 272 Fig. 3. Both TCM decoders invoke the symbol-based MAP 273 algorithm [17] operating in the logarithmic domain. The TCM 274 decoders are labeled with the round-bracketed indexes, whereas 275 the notation $P, A$, and $E$ denote the logarithmic probabilities 276 of the a posteriori, a priori, and extrinsic information, respec277 tively, where $L_{e}=L\left(b_{2} \mid b_{1}\right)$. The $2^{(m+1)}$-ary $P$ probabilities associated with a specific $(m+1)$-bit TTCM-coded symbol 278 $\left\{c_{1}^{\prime}\right\}$ are fed into the TTCM MAP decoder. A pair of signal 279 components is generated by the constituent TCM decoders [17]; 280 specifically, the extrinsic probability $E$ is generated by each 281 of the TCM decoders, whereas the a priori probability $A$ is 282 gleaned by each TCM decoder from the other one. Furthermore, 283 as shown in Fig. 3, the additional extrinsic probability $E_{2} 284$ extracted from the side information $\left\{b_{2}\right\}$ is also added to 285 the a priori probability $A$, yielding $A^{(1,2)}=\left[E_{1}\right]^{(2,1)}+E_{2} .286$ Each of the constituent TCM blocks in Fig. 3 calculates the 287 a posteriori probabilities using the forward and backward re- 288 cursion methods. 4

Upon recalling (4), we are now in the position to formulate 290 the channel's transition metric as

$$
\eta_{i}(\grave{s}, s)=\ln \left\{\frac{1}{\pi N_{0}} \mathrm{e}^{-\left|y_{1}-h x_{1}\right|^{2} / N_{0}}\right\} .
$$

Then, both the backward and forward recursion methods of 292 [17] are invoked for calculating $\beta_{i-1}(\grave{s})$ and $\alpha_{i}(s)$ as follows: 293

$$
\begin{aligned}
\alpha_{i}(s) & =\max _{\operatorname{all} \grave{s}}^{*}\left(\alpha_{i-1}(\grave{s})+\eta_{i}(\grave{s}, s)+A^{(1,2)}\right) \\
\beta_{i-1}(\grave{s}) & =\max _{\text {all } s}^{*}\left(\beta_{i}(s)+\eta_{i}(\grave{s}, s)+A^{(1,2)}\right)
\end{aligned}
$$

where $\max ^{*}$ represents the Jacobian logarithm [17] evaluating 294 all variables in the logarithmic domain, with $(\grave{s}, s)$ denoting the 295 transitions emerging from the previous state $\grave{s}$ to the present 296 state $s$. 


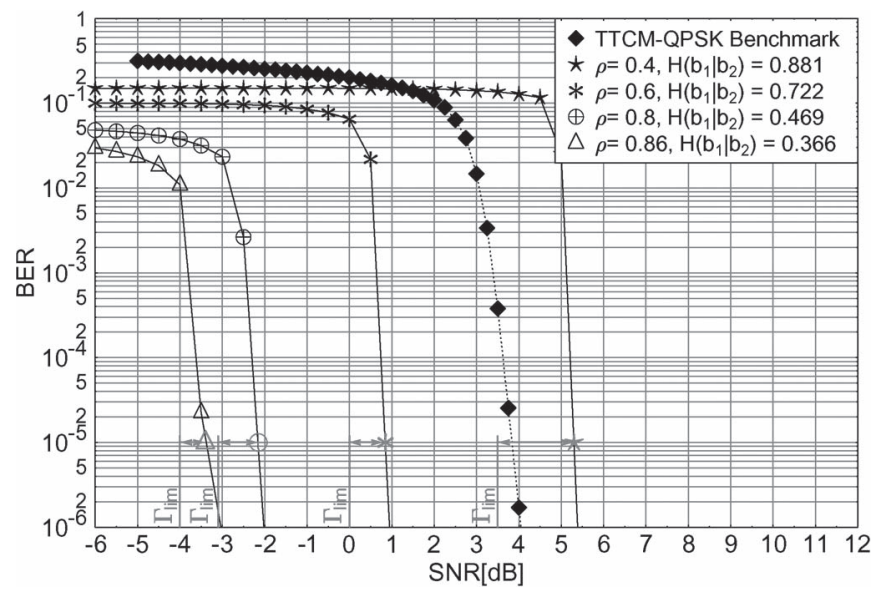

Fig. 4. BER versus SNR performance of the proposed DSTTCMQPSK/BPSK scheme for correlation parameters of $\rho=\{0.4,0.6,0.8,0.86\}$ when transmitting over uncorrelated Rayleigh fading channels. The number of decoding iterations is $I=8$.

\section{RATE REgION DESIGN AND ANALYSiS}

299 The achieved rate region experienced in a noisy channel for 300 both sources is given by [13], [16]:

$$
\begin{aligned}
H\left(b_{1}, b_{2}\right) & \leq \frac{C_{1}}{R_{1}}+\frac{C_{2}}{R_{2}} \\
& \leq \frac{1}{R_{1}} E\left\{\log _{2}\left(1+\gamma_{1}\right)\right\}+\frac{1}{R_{2}} E\left\{\log _{2}\left(1+\gamma_{2}\right)\right\}
\end{aligned}
$$

301 where $C_{1}=E\left\{\log _{2}\left(1+\gamma_{1}\right)\right\}$ and $C_{2}=E\left\{\log _{2}\left(1+\gamma_{2}\right)\right\}$ de302 note the ergodic channel capacities between each of the sources 303 and the destination, whereas $\gamma_{1}$ and $\gamma_{2}$ denote the correspond$304 \mathrm{ing}$ received SNRs. In our asymmetric system, we assume 305 that $\left\{b_{2}\right\}$ is transmitted at $R_{2}=H\left(b_{2}\right)=1$, whereas we aim 306 for compressing $\left\{b_{1}\right\}$ to its minimum rate, namely, to $R_{1}=$ $307 H\left(b_{1} \mid b_{2}\right)$. Then, based on (9), the effective throughput of our 308 scheme for the $\left\{b_{1}\right\}$ link can be expressed as $\eta_{S W}=R_{1}$. $309 H\left(b_{1} \mid b_{2}\right)$, whereas the SW/S bound is calculated as [15]

$$
R_{1} \cdot H\left(b_{1} \mid b_{2}\right) \leq C_{1}
$$

310 where $C_{1}$ represents the ergodic capacity of the uncorrelated 311 Rayleigh fading channel.

312 First we characterize the BER performance of our DSTTCM313 QPSK/BPSK scheme employing a range of correlations $\rho=$ $314\{0.4,0.6,0.8,0.86\}$. We opted for using 1/2-rate TTCM for 315 encoding a block of $N_{S}=12000$ symbols, resulting in $N_{b}=$ 31624000 bits before we remove all of the systematic bits from 317 the TTCM-coded sequence with the aid of puncturing. The 318 BER versus SNR performance of the proposed system is shown 319 in Fig. 4. Note that the SNR can be calculated in decibels 320 as $\operatorname{SNR}(\mathrm{dB})=E_{b} / N_{0}(\mathrm{~dB})+10 \log \left(R_{1}\right)$. The minimum SNR $321 \Gamma_{\lim }$ required for approaching the SW/S bound can be inferred 322 in Fig. 5, which shows both the continuous-input-continuous323 output memoryless channel's (CCMC) capacity and the 324 corresponding BPSK-based discrete-input-continuous-output 325 memoryless channel's (DCMC) capacity curve. For example, 326 when aiming for a target throughput of $\eta_{S W}=0.366$ BPS for 327 our DSTTCM-QPSK/BPSK scheme, the DCMC curve indi-

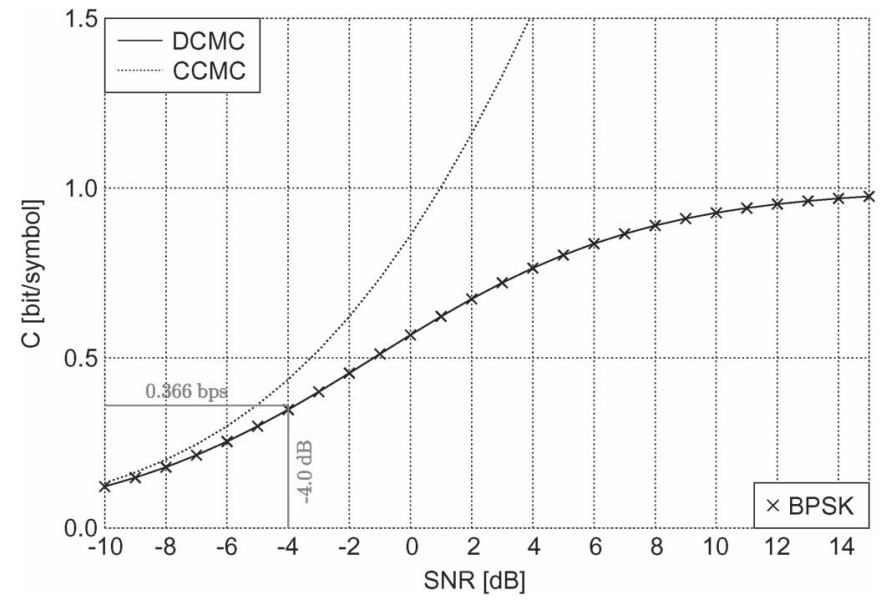

Fig. 5. DCMC and CCMC capacity curves, where the curves were computed based on [24].

cates the requirement of a minimum SNR of $\Gamma_{\lim }=-4 \mathrm{~dB}, 328$ as shown in Fig. 5. Note that $\Gamma_{\lim }$ is represented with the aid of 329 vertical lines in Fig. 4.

As expected, the proposed scheme benefits from the side 331 information constituted by $\left\{b_{2}\right\}$ while compressing the source 332 sequence $\left\{b_{1}\right\}$. Note in Fig. 4 that all DSC schemes outperform 333 the conventional TTCM-QPSK benchmark scheme dispensing 334 with joint decoding, which is labeled by the diamond markers, 335 regardless of the correlation $\rho$, except for the very low correla- 336 tion scenario ${ }^{5}$ of $\rho=0.4$. More explicitly, at a BER $=10^{-5}, 337$ the proposed DSC has an SNR gain of 7.3, 6.2, and $3 \mathrm{~dB} 338$ for $\rho=0.86, \rho=0.8$, and $\rho=0.6$, respectively. However, as 339 expected, with a low correlation value, the proposed scheme 340 has an SNR loss of $1.3 \mathrm{~dB}$, namely, when we have $\rho=0.4$. 341 Again, this is not unexpected because, for $\rho<0.5$, the sources 342 may be deemed to be uncorrelated; hence, they in fact provide 343 misinformation misleading the joint decoder. It may be readily 344 observed in Fig. 4 that, at BER $=10^{-5}$, the scheme having $\rho=345$ 0.86 has the minimum distance with respect to the SW/S limit, 346 i.e., we have $\Gamma-\Gamma_{\lim }=(-3.4)-(-4)=0.6 \mathrm{~dB}$, whereas the 347 scheme associated with $\rho=0.4$ has a distance of $1.7 \mathrm{~dB}$ from 348 the limit.

The effect of the number of iterations between the TCM 350 decoders in Fig. $3 I$, on the overall DSTTCM-QPSK/BPSK 351 scheme's performance, is shown in Fig. 6. It can be observed 352 that doubling the number of iterations from $I=2$ to $I=4353$ will improve the scheme's performance by $1.5 \mathrm{~dB}$, whereas 354 doubling the complexity further will only enhance the system's 355 performance by $0.5 \mathrm{~dB}$. However, doubling the complexity 356 beyond $I=8$ would not provide any further gain at the cost 357 of increasing the decoding complexity; hence, we invoke eight 358 iterations in our decoder.

The SW theoretical bound and the achievable rates obtained 360 for the proposed DSTTCM-QPSK/BPSK schemes are shown, 361 respectively, in Fig. 7. The rates achieved correspond to a 362 BER of $10^{-5}$, and on average, the system's throughput is only 363 0.088 bits away from the bound shown in Fig. 4. Table I 364 summarized the performance of the proposed scheme.

365

\footnotetext{
${ }^{5}$ The higher the crossover probability $p_{e}$, the lower the correlation between the two sources.
} 


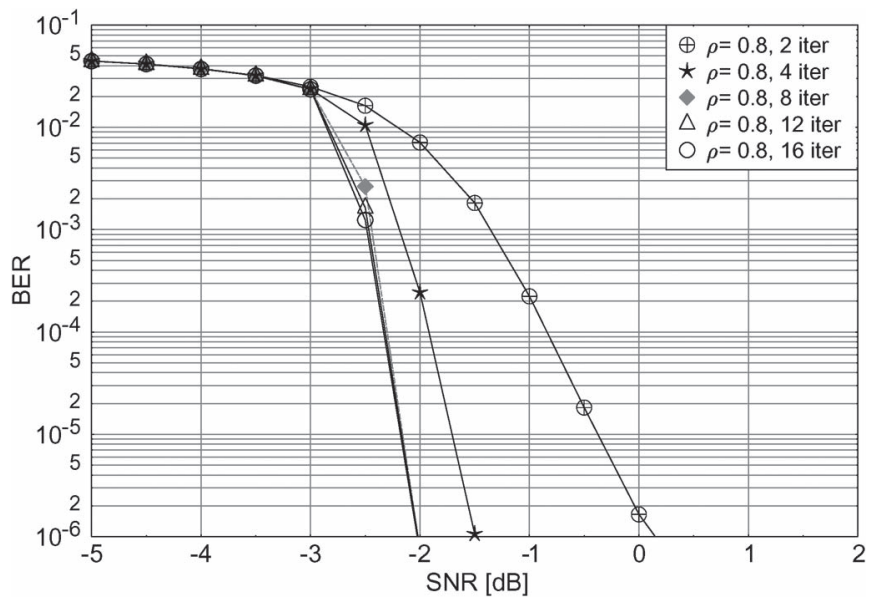

Fig. 6. BER versus SNR performance of the proposed DSTTCMQPSK/BPSK scheme for the correlation parameter of $\rho=\{0.8\}$ when transmitting over uncorrelated Rayleigh fading channels. The number of decoding iterations between the two TCM MAP decoders in Fig. 3 are $I=$ $\{2,4,8,12,16\}$.

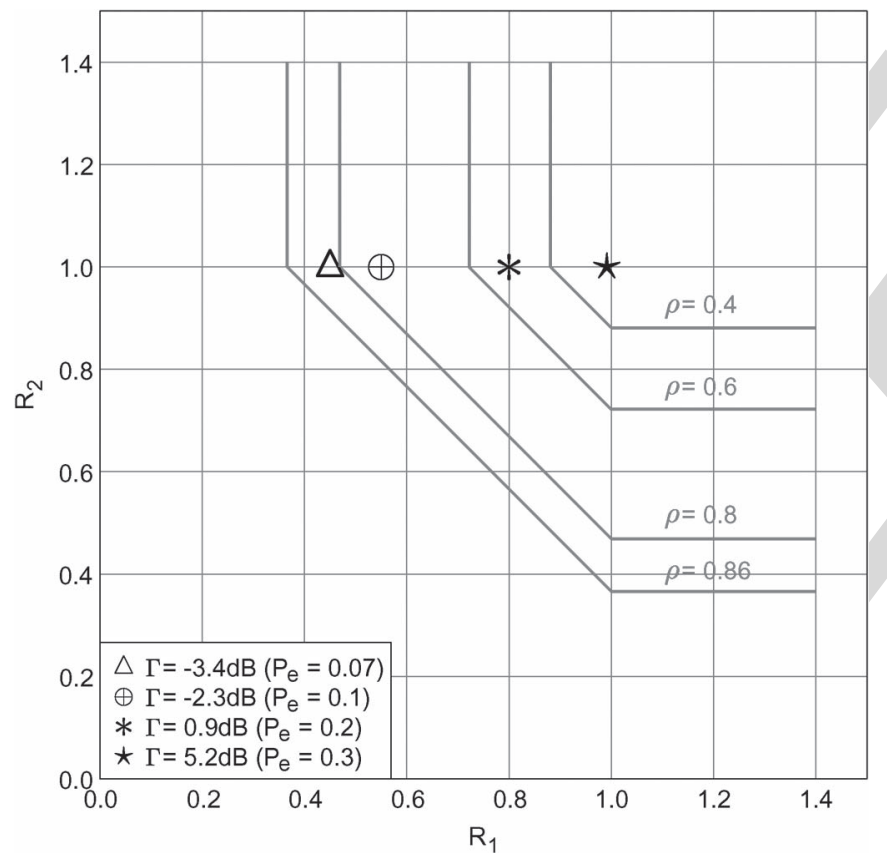

Fig. 7. Theoretical SW bound and the rates $\left(R_{1}, R_{2}\right)$ achieved by the proposed DSTTCM-QPSK/BPSK scheme for different $p_{e}$ values, where $\Gamma$ denotes the SNR required for achieving a BER $=10^{-5}$.

TABLE I

SySTEM PERFOMANCE OF THE DSTTCM FOR SW CODING AT BER $=10^{-5}$

\begin{tabular}{|c|c|c|c|c|c|c|c|}
\hline$p_{e}$ & $\rho$ & $\eta_{S W}$ & $\eta_{10^{-5}}$ & SW-Gap (bits) & $\Gamma_{\lim }$ & $\Gamma$ & Gap (dB) \\
\hline 0.07 & 0.86 & 0.366 & 0.41 & 0.084 & -4.0 & -3.4 & 0.6 \\
\hline 0.1 & 0.80 & 0.469 & 0.55 & 0.081 & -3.1 & -2.3 & 0.80 \\
\hline 0.2 & 0.60 & 0.722 & 0.8 & 0.078 & 0 & 0.9 & 0.90 \\
\hline 0.3 & 0.40 & 0.881 & 1.0 & 0.108 & 3.5 & 5.2 & 1.7 \\
\hline
\end{tabular}

\section{Simulation Results}

367 Our proposed design has also been extended to higher order 368 modulation modes to conceive an adaptive scheme. Fig. 8 369 shows the BER performance of the different DSTTCM modes 370 for $\rho=0.9$ and $\rho=0.8$ associated with crossover probabilities

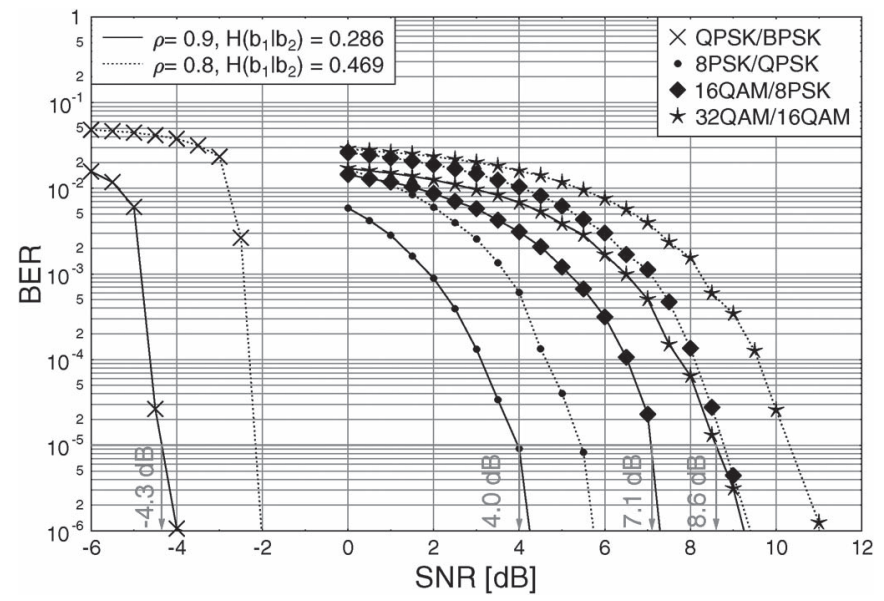

Fig. 8. BER versus SNR performance of the different TTCM modes, when using a block length of $N_{S}=12000$ symbols for $\rho=0.9$ and $\rho=0.86$ for transmission over uncorrelated Rayleigh fading channels.

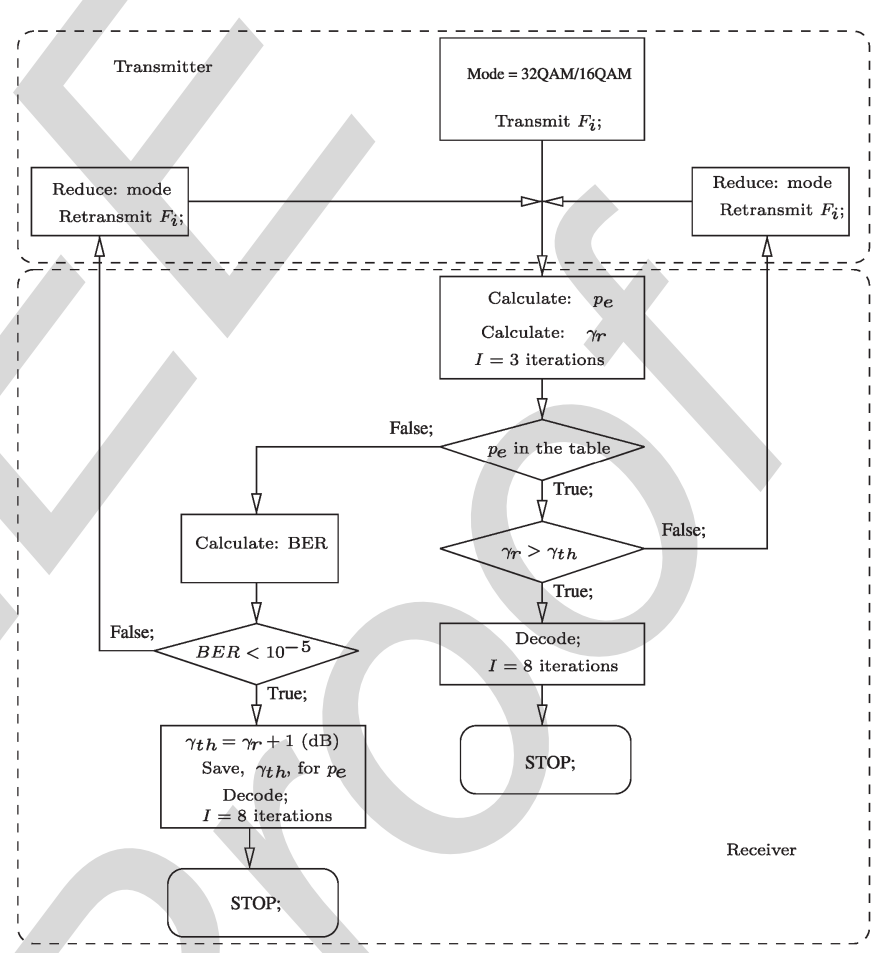

Fig. 9. Flowchart of the adaptive scheme.

of $p_{e}=0.05$ and $p_{e}=0.1$, respectively, while using a block 371 length of $N_{S}=12000$ symbols for all the modulation modes. 372 A total of 10000 blocks have been used in our simulations. 373 The performance of the higher order modulation schemes 374 shown in Fig. 8 suggests that the A-DSTTCM is readily ap- 375 plicable to SW coding. In each mode, we puncture the least 376 significant bit of each coded symbol, which results in punc- 377 turing rates of $R_{p}=\{2 / 1,3 / 2,4 / 3,5 / 4\}$ for QPSK/BPSK, 378 8PSK/QPSK, 16QAM/8PSK, and 32QAM/16QAM, respec- 379 tively. By comparing Figs. 4 and 8, observe that, as expected, 380 the "QPSK/BPSK" scheme outperforms its counterparts in 381 terms of its BER performance since it has a lower throughput. 382

The flowchart in Fig. 9 shows the adaptation process, where 383 the current frame $F_{i}$ will be transmitted first by the highest 384 order 32QAM/16QAM modulation mode. Then, the crossover 385 


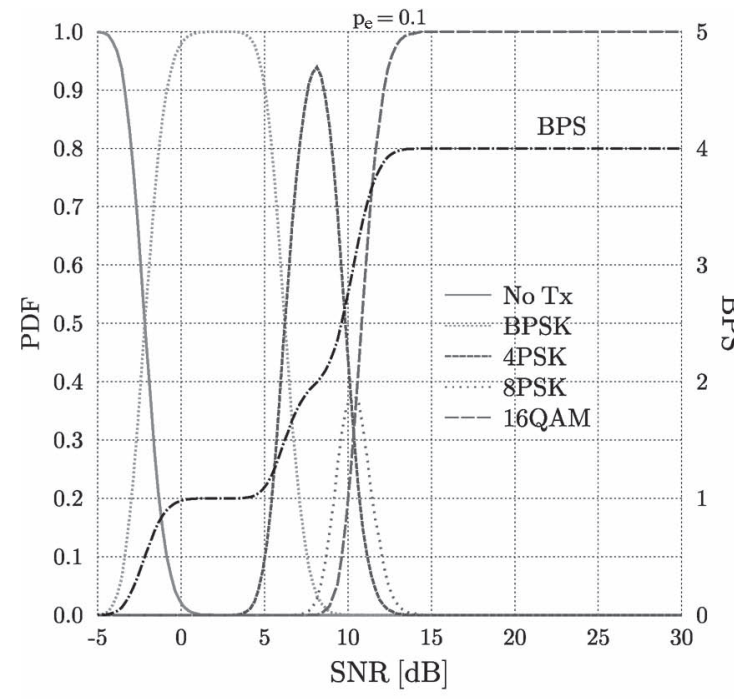

(a)

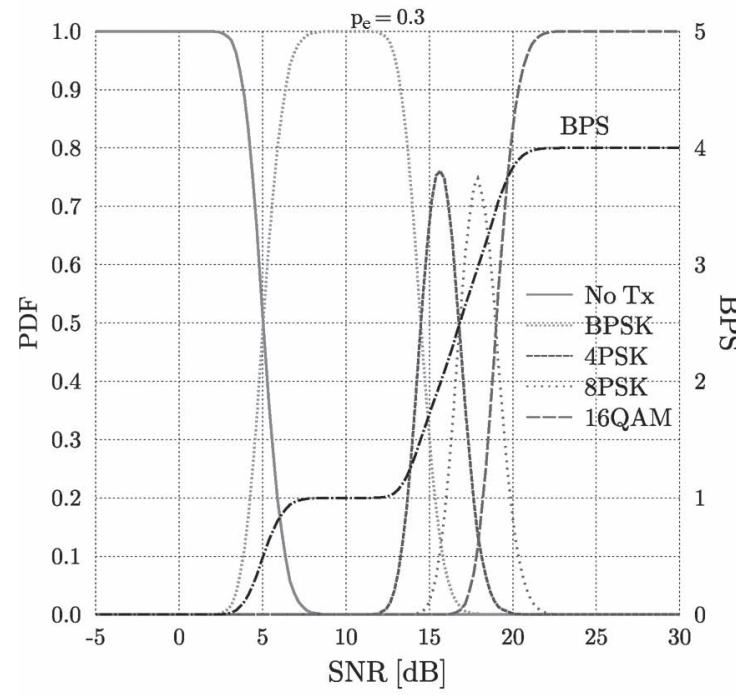

(c)

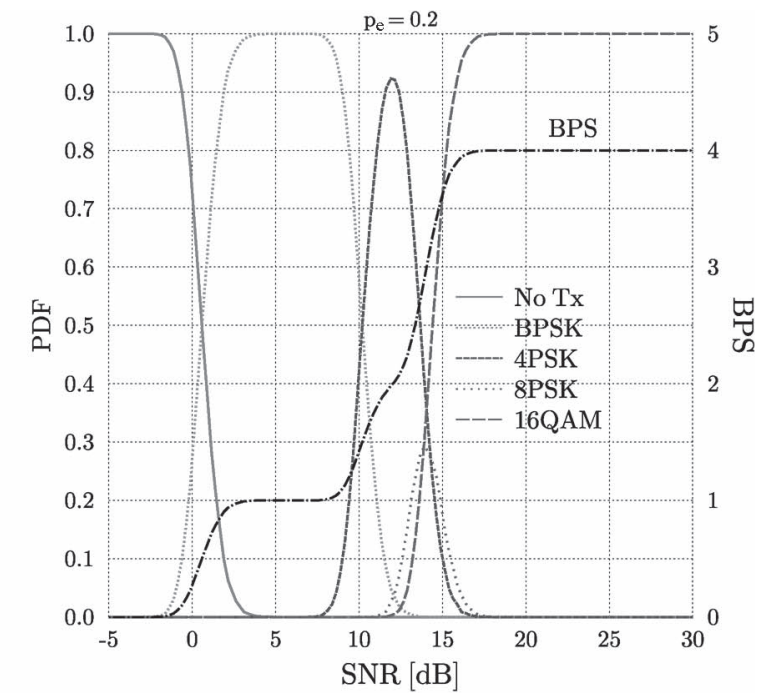

(b)

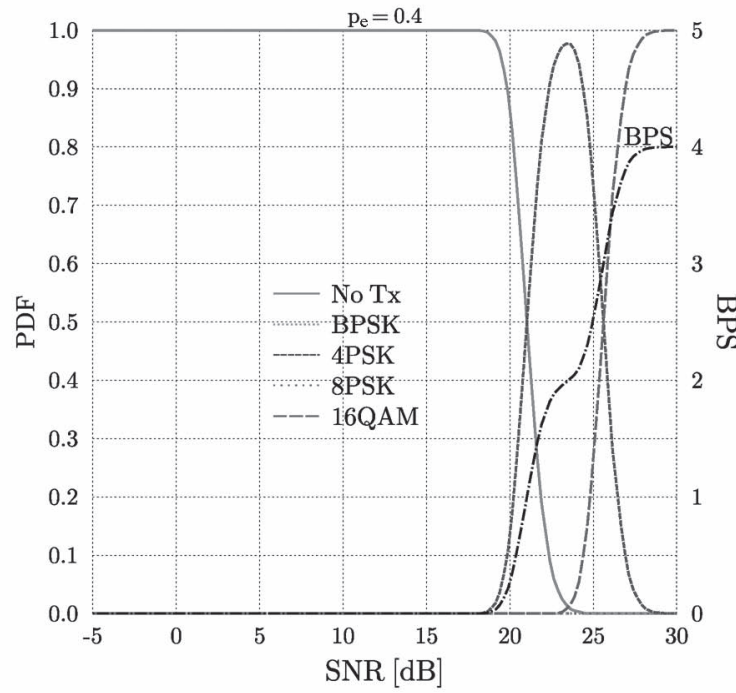

(d)

Fig. 10. PDF of all DSTTCM modes (left-hand vertical axis) versus the received SNR values and the corresponding BPS (right-hand vertical axis) versus the received SNR for $\rho=\{0.8,0.6,0.4,0.2\}$ when communicating over a Rayleigh fading channel. (a) $\rho=0.8$. (b) $\rho=0.6$. (c) $\rho=0.4$. (d) $\rho=0.2$.

386 probability $p_{e}$, which controls the correlation parameter of $\rho=$ $3871-2 p_{e}$, is estimated as [13]

$$
\begin{aligned}
\hat{p}_{e}=\frac{1}{N} \sum_{k=1}^{N} \operatorname{Pr}\left(b_{1}=+1\right) & \operatorname{Pr}\left(b_{2}=-1\right) \\
+ & \operatorname{Pr}\left(b_{1}=-1\right) \operatorname{Pr}\left(b_{2}=+1\right) .
\end{aligned}
$$

388 As few as three iterations are sufficient for estimating the 389 correlation parameter sufficiently accurately. Next, the near390 instantaneous SNR $\gamma_{r}$ is calculated using the following:

$$
\gamma_{r}=10 \log _{10}\left(|h|^{2} / N_{0}\right)[\mathrm{dB}] \text {. }
$$

391 Then, the decoder will compare the estimated probability $\hat{p}_{e}$ to 392 the prestored lookup table. The aim of this table is to reduce 393 the overall complexity of the adaptation process. As shown 394 in the flowchart, if $p_{e}$ is not found in the table, the decoder 395 will estimate the BER of the received block, where the BER is 396 estimated after decoding by comparing the source sequence bits $\left\{b_{1}\right\}$ with the decoded bits $\left\{\hat{b}_{1}\right\}$. Provided that the estimated 397 BER is below $10^{-5}$, the corresponding SNR $\gamma_{r}$, which are 398 indicated by the vertical arrows in Fig. 8 will be saved in the 399 lookup table as the threshold of $\gamma_{\mathrm{th}}=\gamma_{r}+1(\mathrm{~dB})$. Note that 400 the additional $1 \mathrm{~dB}$ and the three iterations used will reassure 401 that the performance of the adaptive scheme will remain below 402 a BER of $10^{-5}$. However, when we have BER $<10^{-5}$ or $\gamma_{r}<403$ $\gamma_{\text {th }}$, a feedback acknowledgement will be transmitted to the 404 transmitter that triggers a reduction of the transmission mode 405 index/throughput. We opt to start with a higher rate mode to 406 reduce the number of feedback requests. ${ }^{6}$ Hence, the overall 407 complexity and time required for performing this adaptive rate 408 adjustment process has been reduced.

409

Furthermore, the probability density function (pdf) of each 410 DSTTCM mode versus the average SNR is shown in Fig. 10, 411 along with the related BPS curves recorded for different 412

\footnotetext{
${ }^{6}$ An incremental mode-based adaptive scheme can be also implemented, subject to additional considerations.
} 
413 correlation of $\rho=\{0.8,0.6,0.4,0.2\}$, respectively. Naturally, 414 the choice of the specific DSTTCM modes is governed by 415 the particular near-instantaneous SNR experienced by the in416 dividual transmission frames at any specific average SNR value 417 in Fig. 10. The adaptive scheme maintains BER $<10^{-5}$. It is 418 shown in Fig. 10 that, as the SNR increases, the higher order 419 DSTTCM modes are activated more often than the lower rate 420 ones. Consequently, the effective BPS throughput increases 421 smoothly with the SNR. It may be also observed from the figure 422 that, in the presence of a high correlation between the two 423 sources, such as $\rho=0.8$, the no transmission mode has effec424 tively disappeared. However, for low correlations, the scheme 425 requires a higher SNR for achieving a nonzero throughput. The 426 adaptive procedure operated as follows. First, the BER of each 427 received block is estimated. If we have BER $<10^{-5}$, then a 428 higher order modulation mode will be requested for the next 429 block.

\section{CONCLUSION}

431 In this paper, we have proposed a novel DSTTCM scheme for 432 a SW-distributed coding system. The proposed system outper433 formed the benchmark scheme dispensing with joint decoding 434 for a wide range of crossover probabilities. A modified symbol435 based MAP was proposed for exploiting the side information 436 available. The theoretical SW/S bounds were estimated, and 437 the proposed scheme was shown to operate within $0.7 \mathrm{~dB}$ 438 from it, for $\rho=0.86$ at a BER of $10^{-5}$. Our proposed scheme 439 operates within 0.088 bits of the maximum achievable rate 440 limit on average, which matches the best result reported in 441 the literature for similar systems while considering a realistic 442 Rayleigh fading channel model. Furthermore, a bandwidth443 efficient practical adaptive scheme was proposed based on a 444 range of adaptive modem modes for transmitting correlated 445 signals over uncorrelated Rayleigh fading channels. The trans446 mitter adapts its coding and modulation modes according to 447 both the short-term channel conditions and to the crossover 448 probabilities for ensuring that the BER remains below $10^{-5}$.

\section{REFERENCES}

[9] J. Garcia-Frias, “Compression of correlated binary sources using turbo 472 codes," IEEE Commun. Lett., vol. 5, no. 10, pp. 417-419, Oct. 2001.473

[10] D. Varodayan, A. Aaron, and B. Girod, "Rate-adaptive codes for dis- 474 tributed source coding," Elsevier Signal Process., vol. 86, no. 11, 475 pp. 3123-3130, Nov. 2006.

[11] A. Liveris, Z. Xiong, and C. Georghiades, "Compression of binary sources 477 with side information at the decoder using LDPC codes," IEEE Commun. 478 Lett., vol. 6, no. 10, pp. 440-442, Oct. 2002.

[12] M. Sartipi and F. Fekri, "Distributed source coding using short to mod- 480 erate length rate-compatible LDPC codes: The entire slepian-wolf rate 481 region," IEEE Trans. Commun., vol. 56, no. 3, pp. 400-411, Mar. 2008.482

[13] J. Garcia-Frias and Y. Zhao, "Near-Shannon/Slepian-Wolf performance 483 for unknown correlated sources over AWGN channels," IEEE Trans. 484 Commun., vol. 53, no. 4, pp. 555-559, Apr. 2005.

[14] Y. Zhao, W. Zhong, and J. Garcia-Frias, "Transmission of correlated 486 senders over a Rayleigh fading multiple access channel," Elsevier Signal 487 Process., vol. 86, no. 11, pp. 3150-3159, Apr. 2006.

[15] J. Del Ser, P. Crespo, and A. Munoz, "Joint source-channel decoding 489 of correlated sources over ISI channels," in Proc. IEEE VTC Spring, 490 Jun. 2005, vol. 1, pp. 625-629.

[16] K. Anwar and T. Matsumoto, "Iterative spatial demapping for two corre- 492 lated sources with power control over fading MAC," in Proc. IEEE VTC 493 Spring, May 2012, pp. 1-7.

[17] L. Hanzo, T. H. Liew, B. L. Yeap, and S. X. Ng, Turbo Coding, Turbo 495 Equalisation and Space-Time Coding: EXIT-Chart Aided Near-Capacity 496 Designs for Wireless Channels. Hoboken, NJ, USA: Wiley, 2010. 497

[18] D. Varodayan, Y.-C. Lin, and B. Girod, "Adaptive distributed source 498 coding," IEEE Trans. Image Process., vol. 21, no. 5, pp. 2630-2640, 499 May 2012.

[19] A. Roumy, K. Lajnef, and C. Guillemot, "Rate-adaptive turbo- 501 syndrome scheme for slepian-wolf coding," in Proc. ACSSC, Nov. 2007, 502 pp. 545-549.

[20] X. Lv, R. Liu, and R. Wang, "A novel rate-adaptive distributed source 504 coding scheme using polar codes," IEEE Commun. Lett., vol. 17, no. 1, 505 pp. 143-146, Jan. 2013.

[21] L. Cui, S. Wang, S. Cheng, and M. Yeary, "Adaptive binary slepian-wolf 507 decoding using particle based belief propagation," IEEE Trans. Commun., 508 vol. 59, no. 9, pp. 2337-2342, Sep. 2011.

509

[22] P. Robertson and T. Wörz, "Bandwidth-efficient turbo trellis-coded mod- 510 ulation using punctured component codes," IEEE J. Sel. Areas Commun., 511 vol. 16, no. 2 , pp. 206-218, Feb. 1998.

[23] V. Toto-Zarasoa, A. Roumy, and C. Guillemot, "Rate-adaptive codes for 513 the entire slepian-wolf region and arbitrarily correlated sources," in Proc. 514 IEEE ICASSP, Apr. 2008, pp. 2965-2968.

[24] S. X. Ng and L. Hanzo, "On the MIMO channel capacity of multi- 516 dimensional signal sets," IEEE Trans. Veh. Technol., vol. 55, no. 2, 517 pp. 528-536, Mar. 2006.

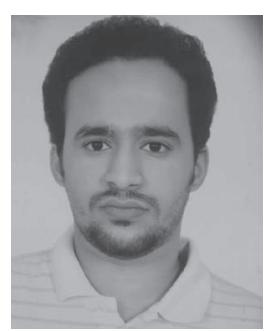

Abdulah Jeza Aljohani received the B.S. degree 519 (with honors) in electronics and communication en- 520 gineering from King Abdulaziz University, Jeddah, 521 Saudi Arabia, in 2006 and the M.Sc. degree (with 522 distinction) in wireless communications from the 523 University of Southampton, Southampton, U.K., in 524 2009. He is currently working toward the Ph.D. 525 degree with the Communications, Signal Processing, 526 and Control Group, School of Electronics and Com- 527 puter Science, University of Southampton. 528

His current research interests include joint source/ 529 channel coding and distributed source coding. 


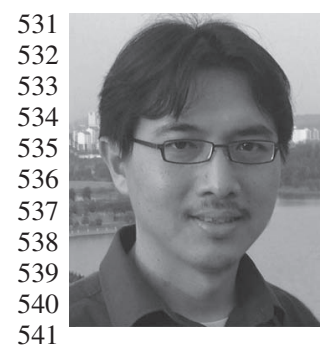

Soon Xin Ng (S'99-M'03-SM'08) received the B.Eng. degree (with first-class honors) in electronics engineering and the Ph.D. degree in wireless communications from the University of Southampton, Southampton, U.K., in 1999 and 2002, respectively.

From 2003 to 2006, he was a Postdoctoral Research Fellow working on collaborative European research projects known as SCOUT, NEWCOM, and PHOENIX. Since August 2006, he has been with the School of Electronics and Computer Science, University of Southampton, where he is currently a 542 Senior Lecturer. He is involved in the OPTIMIX and CONCERTO European 543 projects, as well as the IU-ATC and UC4G projects. He is the author of over 544150 papers and the coauthor of two John Wiley/IEEE Press books. His research 545 interests include adaptive coded modulation, coded modulation, channel 546 coding, space-time coding, joint source and channel coding, iterative detection, 547 orthogonal frequency-division multiplexing, multiple-input-multiple-output 548 systems, cooperative communications, distributed coding, quantum error549 correcting codes, and joint wireless-and-optical-fiber communications.

550 Dr. $\mathrm{Ng}$ is a Chartered Engineer and a Fellow of the Higher Education 551 Academy in the U.K.

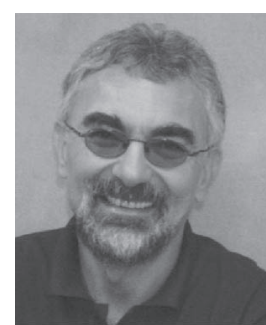

Lajos Hanzo (F'08) received the M.S. degree in 552 electronics, the D.Sc. degree, and the Doctor Honoris 553 Causa degree from the Technical University of Bu- 554 dapest, Budapest, Hungary, in 1976, 1983, and 2009, 555 respectively.

556

During his 37-year career in telecommunications, 557 he has held various research and academic posts in 558 Hungary, Germany, and the U.K. From 2008 to 2012, 559 he was a Chaired Professor with Tsinghua Univer- 560 sity, Beijing, China. Since 1986, he has been with 561 the School of Electronics and Computer Science, 562 University of Southampton, Southampton, U.K., where he is currently the Chair 563 of telecommunications. He is the coauthor of 20 John Wiley/IEEE Press books 564 on mobile radio communications, totalling in excess of 10000 pages, and the 565 author of more than 1300 research entries at IEEE Xplore. He has successfully 566 supervised more than $80 \mathrm{Ph} . \mathrm{D}$. students. He is an enthusiastic supporter of 567 industrial and academic liaison, and he offers a range of industrial courses. 568 Currently, he is directing a 100-strong academic research team, working on a 569 range of research projects in the field of wireless multimedia communications 570 sponsored by industry, the Engineering and Physical Sciences Research Council 571 U.K., the European Research Council's Advanced Fellow Grant, and the 572 Royal Society's Wolfson Research Merit Award. His research is funded by 573 the European Research Council's Senior Research Fellow Grant. For further 574 information on research in progress and associated publications, see (http:// 575 www-mobile.ecs.soton.ac.uk). He has $17000+$ citations.

Dr. Hanzo is a Fellow of the Royal Academy of Engineering, the Institution 577 of Engineering and Technology, and the European Association for Signal 578 Processing. He has served both as a Technical Program Committee and General 579 Chair of IEEE conferences, has presented keynote lectures, and has been 580 awarded a number of distinctions. He is also a Governor of the IEEE Vehicular 581 Technology Society. 
AUTHOR QUERY

NO QUERY.

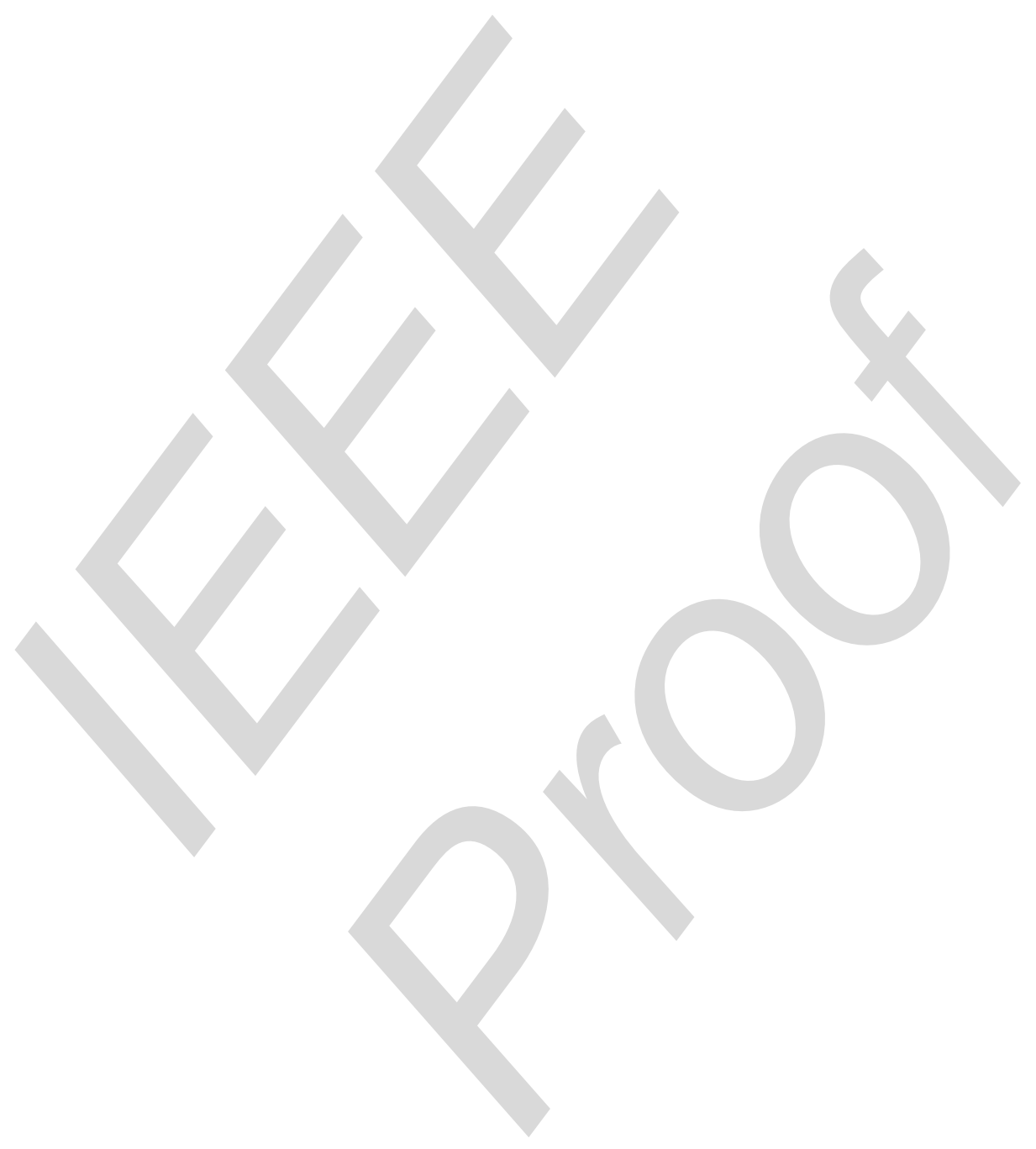

